\title{
Detailed study of the QCD Equation of State of a colorless partonic plasma in finite volume
}

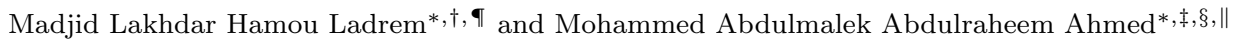 \\ * Physics Department, Faculty of Science, Taibah University, \\ Al-Madinah Al-Munawwarah, Saudi Arabia \\ † Laboratoire de Physique et de Mathématiques et Applications (LPMA), \\ ENS-Vieux Kouba (Bachir El-Ibrahimi), Algiers, Algeria \\ ${ }^{\ddagger}$ Laboratory of Computational Sciences and Mathematical Physics, \\ Institute for Mathematical Research, Universiti Putra Malaysia, \\ 43400 Serdang, Selangor, Malaysia \\ $\S$ Physics Department, Faculty of Science, \\ Taiz University Al-Turba Branch, Taiz, Yemen \\ ฯmladrem@yahoo.fr \\ ॥ mohammed_qgp@taiz.edu.ye \\ Salah Cherif \\ Physics Department, Ghardaia University, Ghardaia, Algeria \\ Laboratoire de Physique et de Mathématiques et Applications (LPMA), \\ ENS-Vieux Kouba (Bachir El-Ibrahimi), Algiers, Algeria \\ salah.cherif.sk@gmail.com \\ Zainab Zaki Mohammed Alfull** and Mosleh M. Almarashi ${ }^{\dagger \dagger}$ \\ Physics Department, Faculty of Science, Taibah University, \\ Al-Madinah Al-Munawwarah, Saudi Arabia \\ **zz-alfull@hotmail.com \\ $\dagger^{\dagger \dagger}$ al_marashi@hotmail.com
}

Received 24 December 2018

Revised 4 March 2019

Accepted 22 March 2019

Published 11 April 2019

The color confinement in Quantum Chromodynamics (QCD) remains an interesting and intriguing phenomenon. It is considered as a very important nonperturbative effect to be taken into account in all models intended to describe the QCD many-parton system.

ฯ Corresponding author.

This is an Open Access article published by World Scientific Publishing Company. It is distributed under the terms of the Creative Commons Attribution 4.0 (CC-BY) License. Further distribution of this work is permitted, provided the original work is properly cited. 


\begin{abstract}
During the deconfinement phase transition, the non-Abelian character of the partonic plasma manifests itself in an important manner. A direct consequence of color confinement is that all states of any partonic system must be colorless and the requirement of the colorlessness condition is more than necessary. Indeed, the colorless state is a result of the multiparton interactions, from which collective phenomena can emerge, inducing strong correlations and giving rise to a long-range order of liquid-like phase, a behavior fundamentally different from that of a conformal ideal gas. Within our Colorless QCD MIT-Bag Model and using the $\mathscr{L}_{m, n}$-method, three Thermal Response Functions, related to the Equation of State, like pressure $\mathscr{P}(T, V)$, sound velocity $\mathscr{C}_{s}^{2}(T, V)$ and energy density $\epsilon(T, V)$ are calculated and studied as functions of temperature $(T)$ and volume $(V)$. Also and in the same context, two relevant correlation forms $\frac{\mathscr{P}}{\epsilon}(\epsilon)$ and $\mathscr{C}_{s}^{2}(\epsilon)$ are calculated and studied intensively as functions of $(\epsilon)$ at different volumes. A detailed comparative study between our results and those obtained from lattice QCD simulation, hot QCD and other phenomenological models is carried out. We find that the Liquid Partonic Plasma Model is the model which fits our Equation of State very well, in which the Bag constant term is revealed very important. Our Colorless Partonic Plasma, just beyond the finite volume transition point, is found in a state where the different partons interact strongly showing a liquid behavior in agreement with the estimate of the plasma parameter $7.92 \leq \Gamma_{\mathrm{CPP}} \leq 10.10$ and supporting the result obtained from the fitting work. This allows us to understand experimental observations in Ultra-Relativistic Heavy-Ion Collisions and to interpret lattice QCD results.
\end{abstract}

Keywords: QGP; colorlessness condition; QCD EoS, nonideal colorless partonic plasma.

PACS numbers: 12.38.Mh, 12.38.Gc, 12.38.Bx, 21.65.Qr, 25.75.Nq

\title{
1. Introduction
}

The Quantum Chromodynamics (QCD) Equation of State (EoS) is of crucial importance for a better comprehension of the strongly interacting matter created in the Ultra-Relativistic Heavy-Ion Collisions (URHIC). ${ }^{4}$ Due to the nonperturbative nature of QCD at low temperatures and small chemical potentials where the system is strongly coupled, the lattice QCD approach is the most successful method for determining the EoS in this part of the phase diagram. However, due to the asymptotic freedom of hot QCD at high temperature, compatibility between the results from lattice QCD calculations and predictions from perturbative theory, where the coupling constant becomes very small, should appear.

It was noted even before the advent of $\mathrm{QCD}^{1,2}$ as the underlying theory of strongly interacting matter that hadronic matter cannot exist as hadrons at an arbitrarily high temperature or density. The existence of a limiting temperature was formulated in the context of the Hagedorn Resonance Gas Model. $\frac{3}{}$ Indeed, in the context of QCD, the existence of a hadronic limiting temperature is synonymous with a phase transition separating ordinary hadronic matter from a new phase of elementary strongly interacting partonic matter namely Quark-Gluon Plasma (QGP) or simply Partonic Plasma (PP). Today, the structure of the phase diagram of the hot $\mathrm{QCD}$ and the determination of the transition point for a system containing two light quarks with a heavy quark are investigated using high precision lattice QCD simulations. 
The properties of the PP are currently under active experimental investigation using the URHIC at the Relativistic Heavy Ion Collider (RHIC) and the Large Hadron Collider (LHC). To make a connection between hot QCD predictions and experimental data, it is essential to formulate a general framework in order to describe the space-time evolution of strongly hot/dense matter produced in URHIC. The Relativistic Hydrodynamics ( $\mathrm{RH}$ ) using appropriate initial conditions is known to be one such framework. The hypothesis of considering dissipative effects beyond the approximation of an ideal PP fluid has been recognized recently both theoretically and experimentally. Among the most important results obtained from the RHIC and LHC experiments, ${ }^{49-52}$ is the fact that the PP was a nearly perfect relativistic fluid, very different from what was supposed to be, a partonic gas, due to the asymptotic freedom of hot QCD. In the case of peripheral collisions, the PP typically has an elliptic shape in the transverse plane. The appearance of spatial azimuthal anisotropy in the momentum distribution is intimately related to the parton-parton interaction. The stronger the parton-parton interaction, the more apparent the asymmetry. Thus, the asymmetry property could indicate that the system is not gas-like. Since the nonideal character of the system, near the transition point even beyond, has been recognized, many attempts have tried to give a plausible explanation for this nonideality by using different models. Such as modified bag models, models with confinement potential, quasiparticle models, strongly interacting PP even we speak about crystal PP model. It is obvious, that in order to understand the physical properties at finite temperature of strongly interacting matter the study of its EoS is of great importance. It highlights the intimate relationship between the degrees of freedom and the different phases of the system. Over the years, we have acquired a solid knowledge concerning this EoS, in the high temperatures regime from perturbation theory and in the low temperatures regime from hadron gas phenomenology. Precisely, in the present work, we want to perform a detailed study of some interesting observables, outshining the physics of the evolution of the EoS of the system undergoing a colorless QCD deconfinement phase transition. At the transition point, the system exhibits, by definition, a singular behavior in some Thermal Response Function (TRF), which appears only in the thermodynamic limit. $\frac{5}{-}$ In both experimental and lattice QCD investigations, we are dealing with finite volume systems. Therefore, they require the development of theoretical approaches that can be able to accurately describe the phase transition in finite-volume including the colorlessness condition. When the volume of the system becomes finite, the singularity is transformed into a finite peak with mathematical properties known as the four finite size effects generating similarities between first-order and second-order phase transitions in finite system. ${ }^{6,9}$ This volume dependence is significant not only in the transition region, but also during the hadronization process. Such an effect has been found, ${ }^{10}$ relating the multiparticle production to the finite volume of the reaction region in an URHIC. 


\section{QCD Equations of State from Some Previous Models}

The pressure of hot QCD, representing one of the most fundamental thermodynamic quantity describing the QCD EoS. It has, under a hard theoretical investigation for several decades now, been computed using a variety of methods and models, including lattice QCD, weak-coupling, and phenomenological models. In order to compare our results concerning the EoS with those obtained the previous models, like lattice QCD simulation, perturbative hot QCD theory or phenomenological models, it is thus noble and honest, to summarize the most relevant results reported in some published articles. The strategy undertaken in this work is to find which model has an EoS integrating the features of our results and reproducing them very well.

\subsection{Hot QCD pressure}

Asymptotic freedom property in QCD theory tells us that strong coupling constant is not really a constant, but that it is a function depending on the energy or momentum scale of the process under investigation. $\frac{1,2}{2}$ In hot QCD, this scale is nothing that the temperature. Since the effective coupling constant $g(T)$ now, in the hot QCD, depends on the temperature, ${ }^{33}$ generating nontrivial thermodynamics and consequently, the partons liberation phenomenon, which would be purely a quantum effect, is possible. In the frame of the hot QCD with the gauge group $S U\left(N_{c}\right)$, and $N_{f}$ flavors of massless quarks, some physicists have computed the pressure at high temperatures in the form of a perturbative expansion in powers of the coupling constant $g(T), \stackrel{34-36}{ }$ known so far up to order $g^{6}(T)$. In the expansion of the pressure, the zeroth-order term represents the pressure of an ideal free partonic gas. The leading term is due to the interaction of order $g^{2}(T)$ which is represented by two-loop diagrams. The leading odd power term of order $g^{3}(T)$ which comes from the one-loop diagram with the resummed static gluon propagator. In our analysis, only the first four terms are considered here,

$$
\begin{aligned}
\frac{\mathscr{P}_{\mathrm{HQCD}}(T)}{T^{4}} & =\mathbf{a}_{\mathrm{QG}}\left(1+\sum_{n=1} \zeta_{n} g^{n}(T)\right) \\
& \approx \mathbf{a}_{\mathrm{QG}}\left(1+\zeta_{1} g(T)+\zeta_{2} g^{2}(T)+\zeta_{3} g^{3}(T)\right)
\end{aligned}
$$

with

$$
g^{2}(T)=\frac{24 \pi^{2}}{\left(11 N_{c}-2 N_{f}\right) \operatorname{Ln}\left(T / \Lambda_{T}\right)}=4 \pi \alpha_{S}(T)
$$

and

$$
\left\{\begin{array}{l}
\zeta_{1}=0 \\
\zeta_{2}=-\frac{5}{2 \pi^{2}} \frac{\left(12+5 N_{f}\right)}{\left(32+21 N_{f}\right)} \\
\zeta_{3}=\frac{20}{\sqrt{6} \pi^{3}} \frac{\left(6+N_{f}\right)^{3 / 2}}{\left(32+21 N_{f}\right)}
\end{array}\right.
$$


The factor $\mathbf{a}_{\mathrm{QG}}$ represents the partonic degrees of freedom (pdof) in the ideal gas state and $\Lambda_{T}$ is the famous QCD scale parameter. If we consider the function $g(T)$ as depending only on the free parameter $\Lambda_{T}$, as an extra parameter $\mathbf{D}_{\mathbf{1}}{ }^{-1}$, we build the first parametrization containing four parameters,

$$
\frac{\mathscr{P}_{1}(T)}{T^{4}}=\mathbf{A}_{\mathbf{1}}-\mathbf{B}_{\mathbf{1}} \operatorname{Ln}^{-1}\left(\mathbf{D}_{\mathbf{1}} T\right)+\mathbf{C}_{\mathbf{1}} \operatorname{Ln}^{-3 / 2}\left(\mathbf{D}_{\mathbf{1}} T\right),
$$

which will be used later in our comparison study.

\subsection{Liquid Partonic Plasma Model Pressure}

The fine analysis of data from hot lattice QCD at $\mu=0$ has yielded very important results which can be described in terms of ideal quantum gases allowing only for lowest order perturbative interactions, provided that some nonperturbative effect is introduced. The agreement is better. This is like in the MIT bag model where a Bag constant is injected in the EoS of the system. We know from the thermal field theory that the whole quantities studied at zero temperature become dependent on the temperature variable. In this context the hot QCD running coupling constant $\alpha_{s}(\mu)$ and the Bag constant $\mathfrak{B}$ should be replaced by a thermal QCD running constant $\alpha_{s}(T)$ and the thermal Bag constant $\mathfrak{B}(T)$. In their papers, ${ }^{37,38}$ Rafelski et al. have taken another approach, combining the hot QCD perturbative result with a nonperturbative $\alpha_{s}(T)$. The EoS of their liquid PP model agrees well with the lattice hot QCD results including the Bag constant except in the vicinity of the deconfinement phase transition point where the nonperturbative behavior of the system is somewhere very important. In the case of $N_{f}$ flavors, their result can be rewritten in the following form:

$$
\frac{\mathscr{P}_{\mathrm{LPPM}}(T)}{T^{4}}=-\frac{\mathfrak{B}}{T^{4}}+\mathbf{a}_{\mathrm{QG}}-\left(\frac{12+5 N_{f}}{18}\right) \pi \alpha_{s}(T)
$$

with the empirical law obtained when the chemical potential of quarks $\mu \rightarrow 0,37,38$

$$
\alpha_{s}(T)=\frac{0.47}{\left(1+0.72 \operatorname{Ln}\left(T / T_{0}\right)\right)} .
$$

The first term is the vacuum contribution and the second term is an ideal gas contribution, with the factor of the pdof in the PP. The third term is the first correction due to the interactions and is exactly the same as the third term given by the perturbative expansion (1). The fourth term, not included in (5), which involves the electric screening mass, and arises from summing an infinite set of diagrams sometimes known as the plasmon diagrams. Finally, we use the same form as in (5) to write our second parametrization,

$$
\frac{\mathscr{P}_{2}(T)}{T^{4}}=\mathbf{A}_{\mathbf{2}}-\frac{\left(\mathbf{B}_{\mathbf{2}}\right)^{4}}{T^{4}}+\frac{\mathbf{C}_{\mathbf{2}}}{\left(1+\mathbf{D}_{\mathbf{2}} \operatorname{Ln}\left(T / T_{0}(V)\right)\right.},
$$

where $T_{0}(V)$ represents the finite volume transition temperature as obtained in our paper. $\cdot$ At high temperature, the above parametrization of the pressure can be 
approximated as,

$$
\begin{aligned}
{\left[\frac{\mathscr{P}_{2}(T)}{T^{4}}\right]_{\mathrm{HT}}=} & \mathbf{A}_{\mathbf{2}}-\frac{\left(\mathbf{B}_{\mathbf{2}}\right)^{4}}{T^{4}}+\frac{\mathbf{C}_{\mathbf{2}} \mathbf{D}_{\mathbf{2}}^{-\mathbf{1}}}{\left(\operatorname{Ln}\left(T / T_{0}(V)\right)\right.} \\
& -\frac{\mathbf{C}_{\mathbf{2}} \mathbf{D}_{\mathbf{2}}^{-\mathbf{2}}}{\left(\operatorname{Ln}\left(T / T_{0}(V)\right)^{2}\right.}+\mathcal{O}\left(\frac{1}{\operatorname{Ln}\left(T / T_{0}(V)\right)^{3}}\right),
\end{aligned}
$$

which should be compared with the relation (4), showing a different exponent of the last term and the presence of the Bag constant term.

\subsection{QCD like potential model pressure}

Bannur in his paper $\stackrel{39,40}{ }$ has derived an EoS for a nonideal PP in which the partons are not free but interacting via QCD like potential (Coulomb + linear confinement). He approached the problem using Mayer's cluster expansion method with the Cornell potential. He obtained an analytical compact expression for the EoS which reproduces the QCD lattice results reasonably well and may be useful in other analytical calculations. This expression is given by

$$
\left(\frac{\mathscr{P}_{P M}(T)}{T^{4}}\right)_{i=G, Q}=\mathbf{a}_{i}-\frac{\mathbf{b}_{i} T^{2}+\mathbf{c}_{i} T+\mathbf{d}_{i}}{\sqrt{\mathbf{e}_{i} T^{4}+\mathbf{f}_{i} T^{3}}} .
$$

We notice that the two expressions (9), in both cases of a pure gluon plasma $G$ and a quark-antiquark plasma $Q$, are similar and the difference resides in numerical values of the six parameters $\left(\mathbf{a}_{i}, \mathbf{b}_{i}, \mathbf{c}_{i}, \mathbf{d}_{i}, \mathbf{e}_{i}, \mathbf{f}_{i}\right)$ which are given in Refs. 39 and 40. Based on the form of the expression (9), the third parametrization, with six parameters, is thereby given by

$$
\frac{\mathscr{P}_{3}(T)}{T^{4}}=\mathbf{A}_{\mathbf{3}}-\frac{\mathbf{B}_{\mathbf{3}}+\mathbf{C}_{\mathbf{3}} T^{-1}+\mathbf{D}_{\mathbf{3}} T^{-2}}{\sqrt{\mathbf{E}_{\mathbf{3}}+\mathbf{F}_{\mathbf{3}} T^{-1}}} .
$$

We have assumed that the expression (9) remains valid to describe the general case of nonideal PP with all multiparton interactions. In high-temperature regime, this relation can be approximated by

$$
\left[\frac{\mathscr{P}_{3}(T)}{T^{4}}\right]_{\mathrm{HT}}=\mathbf{A}_{\mathbf{3}}-\frac{\mathbf{B}_{\mathbf{3}}}{\sqrt{\mathbf{E}_{\mathbf{3}}}}-\frac{\left(2 \mathbf{B}_{\mathbf{3}} \mathbf{D}_{\mathbf{3}}-\mathbf{A}_{\mathbf{3}} \mathbf{E}_{\mathbf{3}}\right) \sqrt{\mathbf{D}_{\mathbf{3}}} \mathbf{A}_{\mathbf{3}}}{2 \mathbf{D}_{\mathbf{3}}{ }^{2}}\left(\frac{1}{T}\right)+\mathcal{O}\left(\frac{1}{T^{2}}\right),
$$

the first two terms represent the ideal partonic gas contribution, which is nothing that the high-temperature limit of the pdof in the PP.

\subsection{Lattice QCD EoS}

Lattice QCD, a gauge theory formulated on finite volume discrete grid of space-time points, is another important approach for the investigation of nonperturbative phenomena such as confinement, which does not admit analytic solutions in quantum field theories. Until now lattice QCD calculations have been successfully conducted in the study of the deconfinement phase transition in all its aspects. All the results 
obtained and published, have been very helpful to understand this deconfinement phase transition and also the thermodynamic properties of the partonic matter. The detailed structure of the phase diagram of the hot QCD and the deconfinement phase transition temperature is becoming more and more accurate regarding the results obtained from calculations using high precision lattice QCD simulations for a system containing two light quarks and a heavy strange quark. From the Refs. $27,28,41$, we noticed that the behavior of the ratio $\mathscr{P} / \epsilon$ at low temperature, and until its minimum point, is consistent with the prediction of a hadron resonance gas model. However, in the high-temperature regime, it has been reported that the ratio $\mathscr{P} / \epsilon$ as a function of the energy density $\epsilon$ can be parametrized with a simple ansatz,

$$
\left(\frac{\mathscr{P}}{\epsilon}\right)_{4}=\frac{1}{3}\left(\mathbf{A}_{4}-\frac{\mathbf{B}_{4}}{\mathbf{C}_{4}+\mathbf{D}_{4} \epsilon}\right)
$$

from which we can easily deduce the parametrization of the sound velocity $\mathscr{C}_{S}^{2}$ as a function of energy density $\epsilon$

$$
\left(\mathscr{C}_{S}^{2}\right)_{4}=\frac{1}{3}\left(\mathbf{A}_{\mathbf{4}}-\frac{\mathbf{E}_{\mathbf{4}}}{\left(\mathbf{C}_{\mathbf{4}}+\mathbf{D}_{\mathbf{4}} \epsilon\right)^{2}}\right) .
$$

This what we call the fourth parametrization.

\section{Colorless QCD MIT-Bag Model: Our Model}

\subsection{Confinement and colorlessness condition}

The confinement phenomenon concerns any many-parton system, and then the colorlessness condition can be considered as an effect of the color interaction between partons rendering the system to be in a colorless state. This manifests itself as a nonideal character in EoS. The conformal ideal gas approximation is quite relevant at high temperature due to the asymptotic freedom in this regime. However, during the transition region, when the system is prepared for colorless deconfinement or just after, things are not so simple, in which the non-Abelian character of the CPP manifests itself in an important manner. Consequently, the consideration of perturbative and nonperturbative corrections of higher order is more than necessary. The colorlessness condition can be then considered as an interaction effect represented by different terms added to the ideal plasma EoS, improving the approximation of the ideal gas and generating an EoS of a nonideal gas. This nonperturbative effect is visible in particular in the transition region. In the vicinity of the transition point, this strong nonperturbative effect dominates the deconfined state. Several models have been proposed to understand this phenomenon, for example, by assuming the appearance of massive quasiparticles, namely massive quarks and gluons. Such a quasiparticle model has also been invoked in solid state physics and other fields to study phase transitions in which a large part of the interaction between the real particles and the medium can be viewed as an effective masses of quasiparticles 
moving freely. In other words, we can say that a system of interacting real particles is equivalent to an ideal quasiparticles system. The calculation of the partition function of the PP without the colorlessness condition means that we take for each possible color state the same probability. But such a trivial approach is inconsistent with the property of color confinement stating that the PP should be colorless. As we are interested in the thermodynamical equilibrium properties of the system, the quantum states of the PP have to comply with that requirement. However, the question remains as to whether it would be possible to explain to how colorless states of partonic system arise in the very early stages of a URHIC.

\subsection{Nonideal plasma and $\Gamma$-parameter}

Collective phenomena emerging from different particle interactions in a many-body system induce strong correlations. These strong correlations are omnipresent in nature that many universal properties observed in strongly correlated systems are explained by the interactions between the building particles. Therefore, the term "correlated behavior" is used to signify deviations from trivial ideal gas behavior. Correlations have a profound effect on the repartition of the particles in phase space. Generally, in a plasma, the motion of a particle is governed by its kinetic energy $\mathscr{E}_{K}$ and potential energy $\mathscr{E}_{P}$, leading to define the ratio between the mean values of the two forms of energy and relate it to the collective behavior of the plasma. This important quantity is called the plasma parameter $\Gamma$ and is defined by,

$$
\Gamma=\frac{\left\langle\mathscr{E}_{P}\right\rangle}{\left\langle\mathscr{E}_{K}\right\rangle} .
$$

In general case, the dimensionless parameter $\Gamma$ as given above can be considered as an order parameter characterizing the transition from a weakly coupled regime with $\Gamma \ll 1$ to a strongly coupled regime with $\Gamma \gg 1$. This parameter can be calculated using relationships derived within different approaches. ${ }^{44,47,48}$ Empirically it is well established for various inter-particle interactions that systems with $\Gamma \leq 100$ are in a liquid phase. For different systems the critical values are different, but they are similar. In our model, the critical value can be taken as: $\Gamma_{c}=1$ for a gas-liquid phase transition. Many previous studies have revealed the different states in which any plasma matter can exist according to the numerical value of the parameter $\Gamma$ : (i) a gas state for $\Gamma \ll 1$, (ii) a liquid state for $\Gamma \approx 10$, (iii) a glass state for $\Gamma \approx 100$, and (iv) a solid state for $\Gamma \gg 300$ (see Refs. 7 and $\underline{8}$ ).

\subsection{Exact colorless partition function}

Due to the color confinement property of QCD, only the colorless part of the parton configurations would manifest themselves as physically observed particles: all many-parton systems are colorless (hadrons, pentaquarks, glueballs, etc.). Therefore, the whole PP fireball needs to be in a colorless state. For this reason, one can consider the QCD deconfinement phase transition as a transition from local 
color confinement $(d \sim 1 \mathrm{fm})$ to global color confinement $(d \gg 1 \mathrm{fm})$. Logically, any theoretical approach which is intended to rigorously describe the QCD deconfinement phase transition must contain colorlessness condition in its formalism. In our previous work and in the context of the Colorless QCD MIT-Bag Model, a new method that allowed us to calculate physical quantities describing the colorless deconfinement phase transition that occurs in a mixed phase system evolving in a finite total volume $V .6,9$ The fraction of volume (defined by the parameter $h$ ) occupied by the $\mathrm{HG}$ phase is given by: $V_{\mathrm{HG}}=h V$, and the remaining volume: $V_{\mathrm{PP}}=(1-h) V$ contains then the CPP phase. If we neglect mutual interactions between the different phases, we can merely write the total partition function of the system as a product of three partition functions:

$$
\mathscr{Z}(h, T, V, \mu)=\mathscr{Z}_{\mathrm{CPP}}(h, T, V, \mu) \mathscr{Z}_{\mathrm{HG}}(h, T, V, \mu) \mathscr{Z}_{\mathrm{Vac}}(h, T, V, \mu),
$$

where

$$
\mathscr{Z}_{\text {Vac }}(h, T, V)=\exp [-(1-h) \mathfrak{B} V / T],
$$

accounts for the difference between the real vacuum and the perturbative vacuum due to the color confinement meaning that the constant $(\mathfrak{B})$ of the bag model represents the pressure on the surface of the bag in order to balance the outward pressure exerted by the partons moving inside the bag. A nonvariable Bag constant $\mathfrak{B}$ is not sufficient because the non-Abelian nature of QCD leads to a complicated nonperturbative structure of QCD vacuum. For the hadronic phase, we simply assumed only pionic degrees of freedom and the corresponding partition function is just given by

$$
\mathscr{Z}_{\mathrm{HG}}(h, T, V)=\exp \mathbf{a}_{\mathrm{HG}} h V T^{3} .
$$

The grand canonical partition function of QCD many-parton system subject to colorlessness condition, by means of the color projection operator $\hat{\mathcal{P}}$, can be written as

$$
\mathscr{Z}_{\mathrm{CPP}}\left(T, V_{\mathrm{PP}}, \mu\right)=\operatorname{Tr}\left(\hat{\mathcal{P}} e^{-\beta(\hat{\mathcal{H}}-\mu \hat{\mathcal{N}})}\right) .
$$

After a considerable amount of algebraic calculations using the group-theoretical projection method ${ }^{11,12}$ and as beautifully performed earlier by Elze et al., ${ }^{13}-18$ one gets the exact colorless partition function for a partonic matter contained in a volume $V_{\mathrm{PP}}$, at a temperature $T$ and quark chemical potential $\mu$, in its final form,

$$
\mathscr{Z}_{\mathrm{CPP}}\left(T, V_{\mathrm{PP}}, \mu\right)=\frac{4}{9 \pi^{2}} \int_{-\pi}^{+\pi} \int_{-\pi}^{+\pi} d \varphi d \psi M(\varphi, \psi) e^{\mathcal{G}\left(\varphi, \psi, \frac{\mu}{T}\right) V_{\mathrm{PP}} T^{3}},
$$

where $M(\varphi, \psi)$ is the weight function (Haar measure) given by

$$
M(\varphi, \psi)=\left[\sin \left(\frac{1}{2}\left(\psi+\frac{\varphi}{2}\right)\right) \sin \left(\frac{\varphi}{2}\right) \sin \left(\frac{1}{2}\left(\psi-\frac{\varphi}{2}\right)\right)\right]^{2}
$$


M. L. H. Ladrem et al.

with

$$
\mathcal{G}\left(\varphi, \psi, \frac{\mu}{T}\right)=\mathcal{G}\left(0,0, \frac{\mu}{T}\right)+\mathcal{G}_{\mathrm{QG}}\left(\varphi, \psi, \frac{\mu}{T}\right) .
$$

The two functions are given in terms of $(T, V, \mu)$ variables as follows:

$$
\mathcal{G}\left(0,0, \frac{\mu}{T}\right)=\mathbf{a}_{\mathrm{QG}}+\frac{N_{f} N_{c}}{6 \pi^{2}}\left(\frac{\mu^{4}}{2 T^{4}}+\frac{\mu^{2} \pi^{2}}{T^{2}}\right)
$$

and

$$
\begin{aligned}
\mathcal{G}_{\mathrm{QG}}\left(\varphi, \psi, \frac{\mu}{T}\right)= & \frac{\pi^{2} N_{c} N_{f}}{36} \sum_{q=r, b, g}\left\{-1+\left(\frac{\left(\alpha_{q}-i\left(\frac{\mu}{T}\right)\right)^{2}}{\pi^{2}}-1\right)^{2}\right\} \\
& -\frac{\pi^{2} N_{g}}{96} \sum_{g=1}^{4}\left(\frac{\left(\alpha_{g}-\pi\right)^{2}}{\pi^{2}}-1\right)^{2}-\frac{N_{f} N_{c}}{6 \pi^{2}}\left(\frac{\mu^{4}}{2 T^{4}}+\frac{\mu^{2} \pi^{2}}{T^{2}}\right) .
\end{aligned}
$$

The two factors $\mathbf{a}_{\mathrm{HG}}$ and $\mathbf{a}_{\mathrm{QG}}$ which are related to the degeneracy number of particles in the system are given by

$$
\left\{\begin{array}{l}
\mathbf{a}_{\mathrm{QG}}=\frac{\pi^{2}}{180}\left(7 N_{c} N_{f}+4 N_{g}\right), \\
\mathbf{a}_{\mathrm{HG}}=\frac{\pi^{2}}{90} N_{\pi},
\end{array}\right.
$$

when $N_{f}, N_{c}, N_{g}$ and $N_{\pi}$ being the number of quark flavors, of color charges, of gluons and of pions, respectively. $\alpha_{q}(q=r, b, g)$ are the angles determined by the eigenvalues of the color charge operators in Eq. (21):

$$
\alpha_{r}=\frac{\varphi}{2}+\frac{\psi}{3}, \quad \alpha_{g}=-\frac{\varphi}{2}+\frac{\psi}{3}, \quad \alpha_{b}=-\frac{2 \psi}{3},
$$

and $\alpha_{g}(g=1, \ldots, 4)$ being $\alpha_{1}=\alpha_{r}-\alpha_{g}, \alpha_{2}=\alpha_{g}-\alpha_{b}, \alpha_{3}=\alpha_{b}-\alpha_{r}, \alpha_{4}=0$. Thus, the partition function of the CPP is then given by

$$
\mathscr{Z}_{\mathrm{CPP}}(h)=\mathscr{Z}_{\mathrm{PP}}(h) \mathscr{Z}_{\mathrm{CC}}(h),
$$

where

$$
\mathscr{Z}_{\mathrm{CC}}(h, T, V, \mu)=\frac{4}{9 \pi^{2}} \int_{-\pi}^{+\pi} \int_{-\pi}^{+\pi} d \varphi d \psi M(\varphi, \psi) e^{(1-h) \mathcal{G}_{\mathrm{QG}}\left(\varphi, \psi, \frac{\mu}{T}\right) V T^{3}}
$$

is the colorless part and,

$$
\mathscr{Z}_{\mathrm{PP}}(h, T, V, \mu)=\exp (1-h) V T^{3} \mathcal{G}\left(0,0, \frac{\mu}{T}\right)
$$

is the PP part without the colorlessness condition. Finally, the exact total colorless partition function is given by

$$
\mathscr{Z}(h, T, V, \mu)=\mathscr{Z}_{0}(h, T, V, \mu) \mathscr{Z}_{\mathrm{CC}}(h, T, V, \mu)
$$


with

$$
\mathscr{Z}_{0}(h, T, V, \mu)=\mathscr{Z}_{\mathrm{HG}}(h, T, V, \mu) \mathscr{Z}_{\mathrm{Vac}}(h, T, V, \mu) \mathscr{Z}_{\mathrm{PP}}(h, T, V, \mu) .
$$

This latter is only the total grand partition function of the system without the colorlessness condition, which can be rewritten in its most familiar form obtained in earliest papers (see for example Ref. 22):

$\operatorname{Ln} \mathscr{Z}_{0}(h, T, V, \mu)=\left[\left(\mathbf{a}_{\mathrm{QG}}+\frac{N_{c} N_{f}}{6 \pi^{2}}\left(\pi^{2} \frac{\mu^{2}}{T^{2}}+\frac{\mu^{4}}{2 T^{4}}\right)-\frac{\mathfrak{B}}{T^{4}}\right)(1-h)+\mathbf{a}_{\mathrm{HG}} h\right] V T^{3}$.

We will investigate some additional consequences of the SU(3) colorlessness condition of partonic matter beyond those studied and published by Elze et al. ${ }^{13-18}$ especially in the context where no approximation is used.

\subsection{Finite size Hadronic Probability Density Function and exact $\mathscr{L}_{m, n}$-method}

The definition of the Hadronic Probability Density Function (HPDF) in our model $^{6,9}$ is given by

$$
p(h, V, T, \mu)=\frac{\mathscr{Z}(h, V, T, \mu)}{\int_{0}^{1} \mathscr{Z}(h, V, T, \mu) d h} .
$$

Since our HPDF is directly related to the partition function of the system, it is believed that the whole information concerning the QCD matter and its colorless deconfinement phase transition is self-contained in this HPDF. Then we can perform the calculation of the mean value of any TRF: $\mathcal{Q}(h, T, \mu, V)$, characterizing the system in the state $h$ by

$$
\mathcal{Q}(T, \mu, V)=\int_{0}^{1} Q(h, T, \mu, V) p(h, V, T, \mu) d h .
$$

It is worth remembering that in our first work, we have used the colorless partition function, as calculated by Elze et al. $\underline{13}-\underline{18}$ without any approximation. The impossibility of calculating the color integrals analytically, compelled us to develop a new method in order to calculate easily physical TRF describing well the colorless deconfinement phase transition in a finite volume $V . \underset{6,19}{ }$ This method will enable us to carry out the study of the finite volume effects on the thermal evolution of the EoS in the context of the Colorless QCD MIT-Bag Model. The EoS of a system undergoing the colorless deconfinement from a hadronic matter described by a hadron mass spectrum including the effects due to the finite hadron eigenvolumes was investigated in detail. ${ }^{20,21}$ Even in this case, the analytical calculation would not have been possible without using the saddle point approximation. In the present work, we will consider the case of vanishing chemical potential $(\mu=0)$ and on only two lightest quarks $u$ and $d\left(N_{f}=2\right)$ and a massless pionic matter. The common value $\mathfrak{B}^{1 / 4}=145 \mathrm{MeV}$ for the Bag constant will be used. 
The most important result consists in the fact that in principle all TRF calculated in this context can be merely expressed as a function of only a certain double integral thermodynamic function $\mathscr{L}_{m, n}(q, T, V)$, which are defined as

$$
\mathscr{L}_{m, n}(q, T, V)=\int_{-\pi}^{+\pi} \int_{-\pi}^{+\pi} d \varphi d \psi M(\varphi, \psi)(\mathcal{G}(\varphi, \psi, 0))^{m} \frac{e^{q \mathscr{R}(\varphi, \psi ; T, V)}}{(\mathscr{R}(\varphi, \psi ; T, V))^{n}},
$$

where the function $\mathscr{R}(\varphi, \psi ; T, V)$ is given by

$$
\mathscr{R}(\varphi, \psi ; T, V)=\left(\mathcal{G}(\varphi, \psi, 0)-\mathbf{a}_{\mathrm{HG}}-\frac{\mathfrak{B}}{T^{4}}\right) V T^{3} .
$$

This method is named the $\mathscr{L}_{m, n}$-method. It is obvious that these $\mathscr{L}_{m, n}(q, T, V)$ are just state functions depending on $(T, V)$ and of course on state variable $q$, and they can be calculated numerically at each temperature $T$ and volume $V$. As we have explained before that the mean value of any physical quantity $\mathcal{Q}(h, T, 0, V)$ can therefore be calculated as a simple function of these $\mathscr{L}_{m, n}(q, T, V)$ evaluated in the hadronic phase $\mathscr{L}_{m, n}(q=0, T, V)$ and in the CPP phase $\mathscr{L}_{m, n}(q=1, T, V)$. An other important property of these $\mathscr{L}_{m, n}(q, T, V)$ coefficients relies on the fact that any derivative within the $(T, V)$ variables giving rise to other $\mathscr{L}_{m, n}(q, T, V)$ coefficients, it is like making a connection between different $\mathscr{L}_{m, n}(q, T, V)$ and combining them in a simple recurrence relation.

By means of $\mathscr{L}_{m, n}$-method, we have developed a new method of calculation which simplify the final mathematical form of any TRF and to make it easier to calculate numerically. Nevertheless, it is helpful to consider how the thermodynamics of the total system is formulated in the context of this $\mathscr{L}_{m, n}$-method.

\section{Finite Volume Colorless QCD Thermodynamics and Equation of State: Results and Discussions}

\subsection{The importance of the EoS in the hydrodynamical description}

Let us remember that the system subject to our study and formed in URHIC starts expanding soon as it is produced. On both sides of the deconfinement phase transition, it contains a huge number of particles in small finite volume (partons in the $\mathrm{PP}$ and hadrons in the $\mathrm{HG}$ ). If these particles interact strongly enough, the system may reach a state of local thermodynamical equilibrium. If it can be locally maintained during the subsequent expansion, the further evolution of the CPP and HG can be described conveniently by $\mathrm{RH}$. Hydrodynamics is a macroscopic approach which describes the system by macroscopic variables, such as local energy density $\epsilon(T, V)$, pressure $\mathscr{P}(T, V)$, sound velocity $\mathscr{C}_{s}^{2}$, the dynamical number of degrees of freedom and entropy density $\mathscr{S}(T, V)$. It requires knowledge of the EoS, which gives a relation between pressure, energy and entropy density, but not detailed knowledge of the microscopic dynamics. This what we want to do by studying these TRF and their correlations. Generally, in the phenomenological approaches of URHIC physics, we always need an EoS to model the hydrodynamic expansion of the system 
of particles formed in these collisions. The space-time pattern of a collision, depends strongly on the type of the EoS, and the phase transition leads to an increase in the collision time. In order to reach a detailed understanding of the EoS describing the strongly interacting matter undergoing the deconfinement phase transition, the detailed study of the temperature dependence of some TRF like: pressure $\mathscr{P}(T, V)$, sound velocity $\mathscr{C}_{s}^{2}(T, V)$ and energy density $\epsilon(T, V)$ as well as $\mathscr{P}(\epsilon)$ and $\mathscr{C}_{s}^{2}(\epsilon)$, is really of great importance both experimentally and theoretically.

\subsection{Some important thermal response functions}

\subsubsection{Two fundamental quantities: Order parameter $\mathscr{H}(T, V)$ and Violette term $\mathcal{V}(T, V)$}

The first quantity of interest in our study was the mean value of the hadronic volume fraction $\mathscr{H}(T, V)$, which is considered as the order parameter for the phase transition investigated in this work. According to (32) and (33), these quantities can be expressed as,, 23

$$
\mathscr{H}(T, V)=\langle h(T, V)\rangle=\int_{0}^{1} h p(h) d h .
$$

We can also define the fraction of the total volume occupied by the PP as $q=1-h$ and its mean value as

$$
\mathscr{Q}(T, V)=\langle q(T, V)\rangle=1-\mathscr{H}(T, V) .
$$

In terms of $\mathscr{L}_{m, n}$ and from (36), we can easily express them as

$$
\begin{gathered}
\mathscr{H}(T, V)=\frac{\mathscr{L}_{0,2}(1)-\mathscr{L}_{0,2}(0)-\mathscr{L}_{0,1}(0)}{\mathscr{L}_{0,1}(1)-\mathscr{L}_{0,1}(0)}, \\
\mathscr{Q}(T, V)=\frac{\mathscr{L}_{0,2}(0)-\mathscr{L}_{0,2}(1)+\mathscr{L}_{0,1}(1)}{\mathscr{L}_{0,1}(1)-\mathscr{L}_{0,1}(0)} .
\end{gathered}
$$

The second one was the mean value of the partonic number of degrees of freedom (pdof) $\mathscr{V}(T, V)$ (called the Violette term), $, 9,23$

$$
\mathscr{V}(T, V)=\frac{\mathscr{L}_{1,1}(1)-\mathscr{L}_{1,2}(1)+\mathscr{L}_{1,2}(0)}{\mathscr{L}_{0,1}(1)-\mathscr{L}_{0,1}(0)} .
$$

Also, we need to define two thermal susceptibilities as the temperature derivatives:

- Hadronic thermal susceptibility

$$
\chi_{T}^{H}(T, V)=\frac{\partial \mathscr{H}(T, V)}{\partial T} .
$$

- Partonic thermal susceptibility

$$
\chi_{T}^{P}(T, V)=\frac{\partial \mathscr{V}(T, V)}{\partial T} .
$$

We will see that the most important physical quantities can be written by means of these fundamental quantities. 


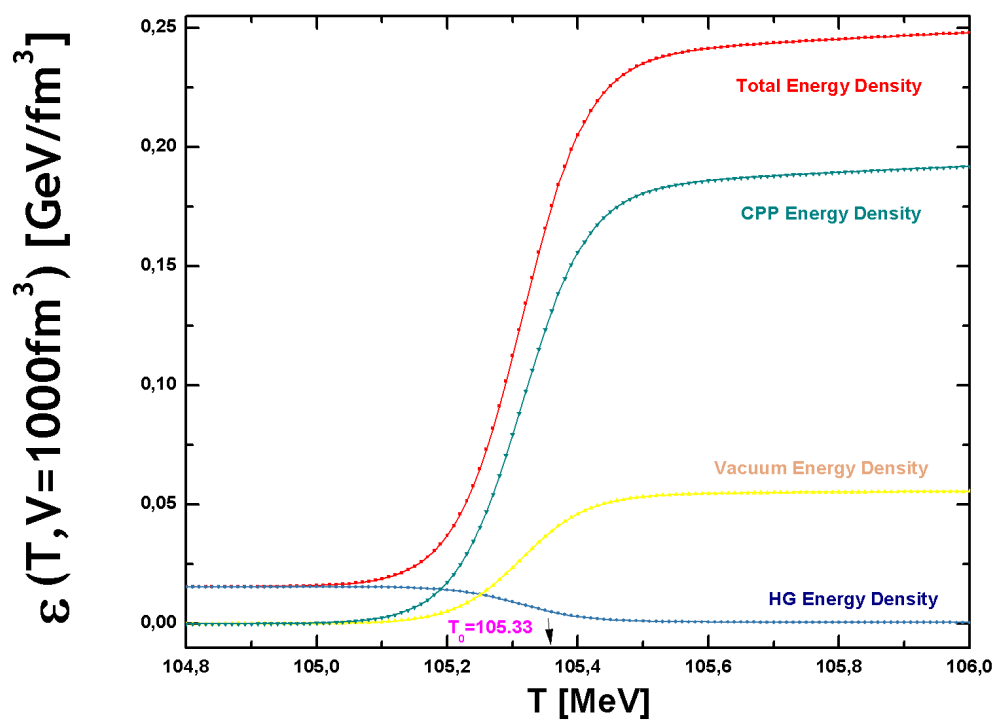

Fig. 1. Different contributions in energy density $\epsilon(T, V)$ versus temperature $T$.

\subsubsection{Energy density: $\epsilon(T, V)$}

The third quantity of interest was the energy density $\epsilon(T, V)$, whose mean value was also calculated in the same way, and was found to be related to $\mathscr{H}(T, V)$ and $\mathcal{V}(T, V)$ by the relationship,

$$
\begin{aligned}
\epsilon(T, V) & =\frac{T^{2}}{V}\left\langle\left(\frac{\partial \operatorname{Ln} \mathscr{Z}(h, T, V)}{\partial T}\right)\right\rangle \\
& =3 \mathbf{a}_{\mathrm{HG}} T^{4} \mathscr{H}(T, V)+\mathfrak{B} \mathscr{Q}(T, V)+3 T^{4} \mathscr{V}(T, V) .
\end{aligned}
$$

We note that this relation has an important form expressing the splitting of the energy density on three different terms representing the contribution of each phase in the system. In Fig. 1, one can clearly see these three contributions at $V=1000 \mathrm{fm}^{3}$. However, the normalized energy density of the system $\frac{\epsilon(T, V)}{T^{4}}$ is represented by the 3 -dim plot in Fig. 2. When we calculate the thermal derivative of the $\epsilon(T, V)$, one finds the specific heat of the system

$$
\begin{aligned}
c_{T}(T, V)= & \left(\frac{\partial \epsilon(T, V)}{\partial T}\right) \\
= & 12 T^{3}\left[\mathbf{a}_{\mathrm{HG}} \mathscr{H}(T, V)+\mathscr{V}(T, V)\right] \\
& +3 T^{4}\left(\mathbf{a}_{\mathrm{HG}} \chi_{T}^{H}+\chi_{T}^{P}\right)-\mathfrak{B} \chi_{T}^{H} .
\end{aligned}
$$

Inserting (38), (39) and (40) in (43) we get the final expression of $\epsilon(T, V)$ in terms of $\mathscr{L}_{m, n}(q, \bar{T}, V)$. 


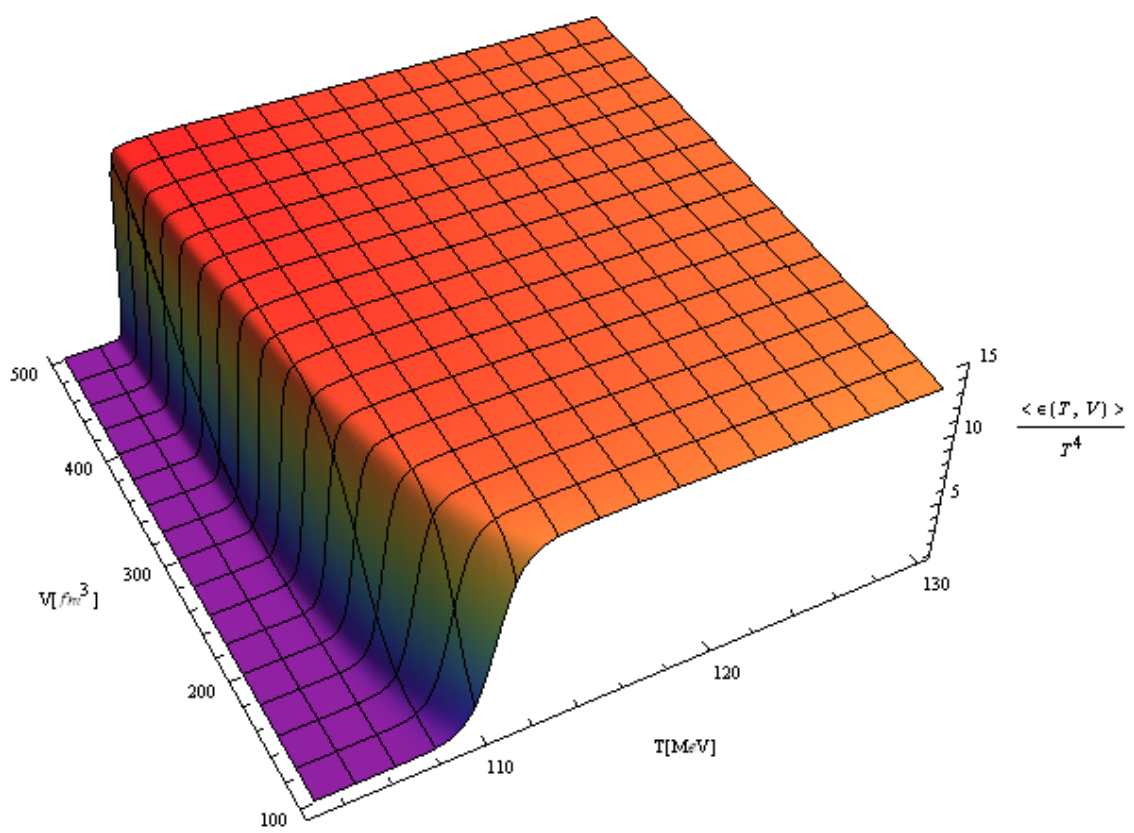

Fig. 2. 3-dim normalized energy density $\frac{\epsilon(T, V)}{T^{4}}$ of the system as a function of $(T, V)$.

The quantity $\frac{\epsilon(T, V)}{T^{4}}$ is traditionally interpreted as a measure of the number of effective degrees of freedom in the system. The natural behavior observed on the 3-dim plot (Fig. 2) of the normalized energy density at the colorless QCD deconfinement phase transition, that is logically compelling, is the liberation/melting of the partonic degrees of freedom frozen in the hadronic phase. This deconfinement gives rise to a finite smooth discontinuity in finite volume becoming a finite sharp discontinuity in the thermodynamic limit, which is related to the latent heat of the first-order colorless deconfinement phase transition. A clear size dependence over almost the entire temperature range is noticed. The finite discontinuity can be mathematically described by a step function, which transforms into a $\delta$-function in the specific heat $c_{T}(T, V)$ and could serve as an indicator for the finite volume transition point.

\subsubsection{Pressure: $\mathscr{P}(T, V)$}

The thermodynamic pressure is calculated using the definition: ${ }^{24}-26$

$$
\mathscr{P}(T, V)=T\left\langle\left(\frac{\partial \operatorname{Ln} \mathscr{Z}(h, T, V)}{\partial V}\right)\right\rangle,
$$

and after a straightforward calculation, we get the final expression showing the contribution of the pressure of each phase contained in our system,

$$
\mathscr{P}(T, V)=\mathbf{a}_{\mathrm{HG}} T^{4} \mathscr{H}(T, V)-\mathfrak{B} \mathscr{Q}(T, V)+T^{4} \mathscr{V}(T, V) .
$$




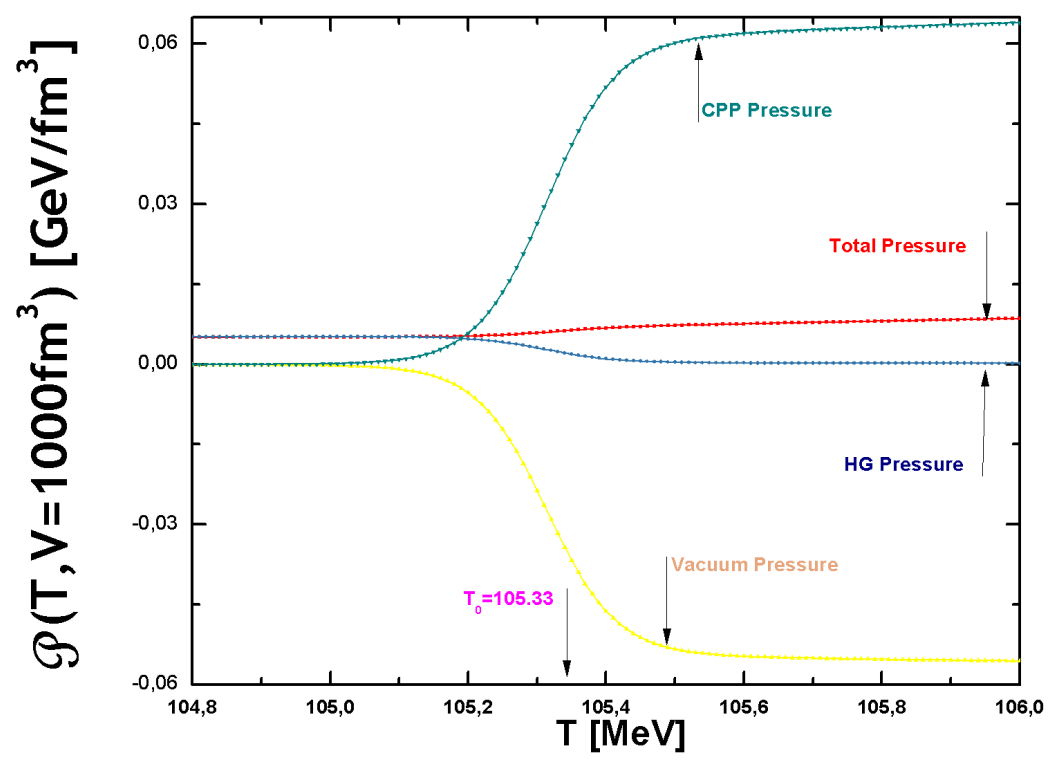

Fig. 3. Different contributions in the pressure $\mathscr{P}\left(T, V=1000 \mathrm{fm}^{3}\right)$ versus temperature $T$.

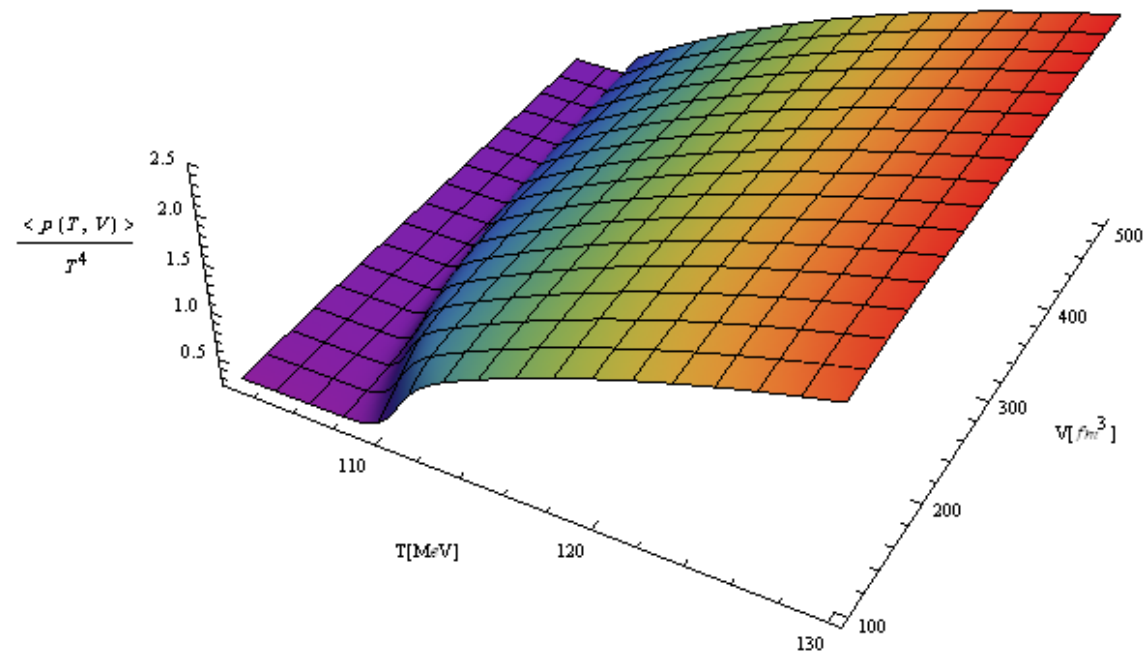

Fig. 4. 3-dim normalized pressure $\frac{\mathscr{P}(T, V)}{T^{4}}$ of the system as a function of $(T, V)$.

Figure 3 displays the three contributions of the pressure in the different phases of the system at $V=1000 \mathrm{fm}^{3}$. Figure 4 shows the 3 -dim plot of the calculated pressure normalized by $T^{4}$. A rapid growth of the pressure is clearly seen just after the transition temperature, especially when the volume approaches the thermodynamic limit. It can be clearly seen that below the transition temperature, the pressure is 
constant with the Stefan-Boltzmann value in HG phase, then it abruptly increases around $T_{0}(V)$ and continues increasing, rather slowly this time. One sees that the pressure undergoes a monotonic increase continuously as a function of temperature. A behavior that is consistent with the phase coexistence phenomenon. When expressing the pressure as a function of the energy density, the relation becomes more simple

$$
3 \mathscr{P}(T, V)=\epsilon(T, V)-4 \mathfrak{B} \mathscr{Q}(T, V) .
$$

Here also, and with the same spirit, we can obtain the expression of $\mathscr{P}(T, V)$ as a function of $\mathscr{L}_{m, n}(q, T, V)$ using (38), (39) and (40).

\subsubsection{Sound velocity: $\mathscr{C}_{s}^{2}(T, V)$}

The squared sound velocity $\mathscr{C}_{s}^{2}(T, V)$, the most relevant measure of the system's tendency to expand when calculated from its definition, is given by

$$
\begin{aligned}
\mathscr{C}_{s}^{2}(T, V) & =\left(\frac{\partial \mathscr{P}}{\partial \epsilon}\right) \\
& =c_{T}^{-1}(T, V)\left(\frac{\partial \mathscr{P}(T, V)}{\partial T}\right) \\
& =\frac{4 T^{3}\left[\mathbf{a}_{\mathrm{HG}} \mathscr{H}(T, V)+\mathscr{V}(T, V)\right]+T^{4}\left(\mathbf{a}_{\mathrm{HG}} \chi_{T}^{H}+\chi_{T}^{P}\right)+\mathfrak{B} \chi_{T}^{H}}{12 T^{3}\left[\mathbf{a}_{\mathrm{HG}} \mathscr{H}(T, V)+\mathscr{V}(T, V)\right]+3 T^{4}\left(\mathbf{a}_{\mathrm{HG}} \chi_{T}^{H}+\chi_{T}^{P}\right)-\mathfrak{B} \chi_{T}^{H}} .
\end{aligned}
$$

This can be written in terms $\mathscr{L}_{m, n}(q, T, V)$ via the two thermal susceptibilities $\chi_{T}^{P}(T, V)$ and $\chi_{T}^{H}(T, V)$. After a straightforward calculation, $\mathscr{C}_{s}^{2}(T, V)$ can be related to both hadronic thermal susceptibility $\chi_{T}^{H}(T, V)$ and specific heat density $c_{T}(T, V)$, by the relation:

$$
\mathscr{C}_{s}^{2}(T, V)=\frac{1}{3}\left[1+4 \mathfrak{B} \frac{\chi_{T}^{H}(T, V)}{c_{T}(T, V)}\right] .
$$

The 3-dim plot in Fig. 5 displays the variation of $\mathscr{C}_{s}^{2}(T, V)$ as a function of temperature and volume. It is obvious from the plot that for temperatures well below and/or above a transition temperature, it approaches the value of an ideal gas: $\mathscr{C}_{s}^{2}=\frac{1}{3}$. In finite volume, the phase transition digs in the plane $\mathscr{C}_{s}^{2}(T, V)=\frac{1}{3}$ a kind of valley whose deepest point is nothing else than the transition point $T_{0}(V)$. Also, we noticed that the clear finite volume scaling law of the position of this point as a function of the volume: $T_{0}(V)-T_{0}(\infty) \propto V^{-1}$ as displayed in Fig. 5. Finite volume thermodynamic observable, such as pressure and sound velocity show deviations from conformal ideal gas behavior even beyond the finite volume transition point $T_{0}(V)$ and approach the ideal gas limit relatively in a rapid way than in the case of the lattice QCD simulation. 


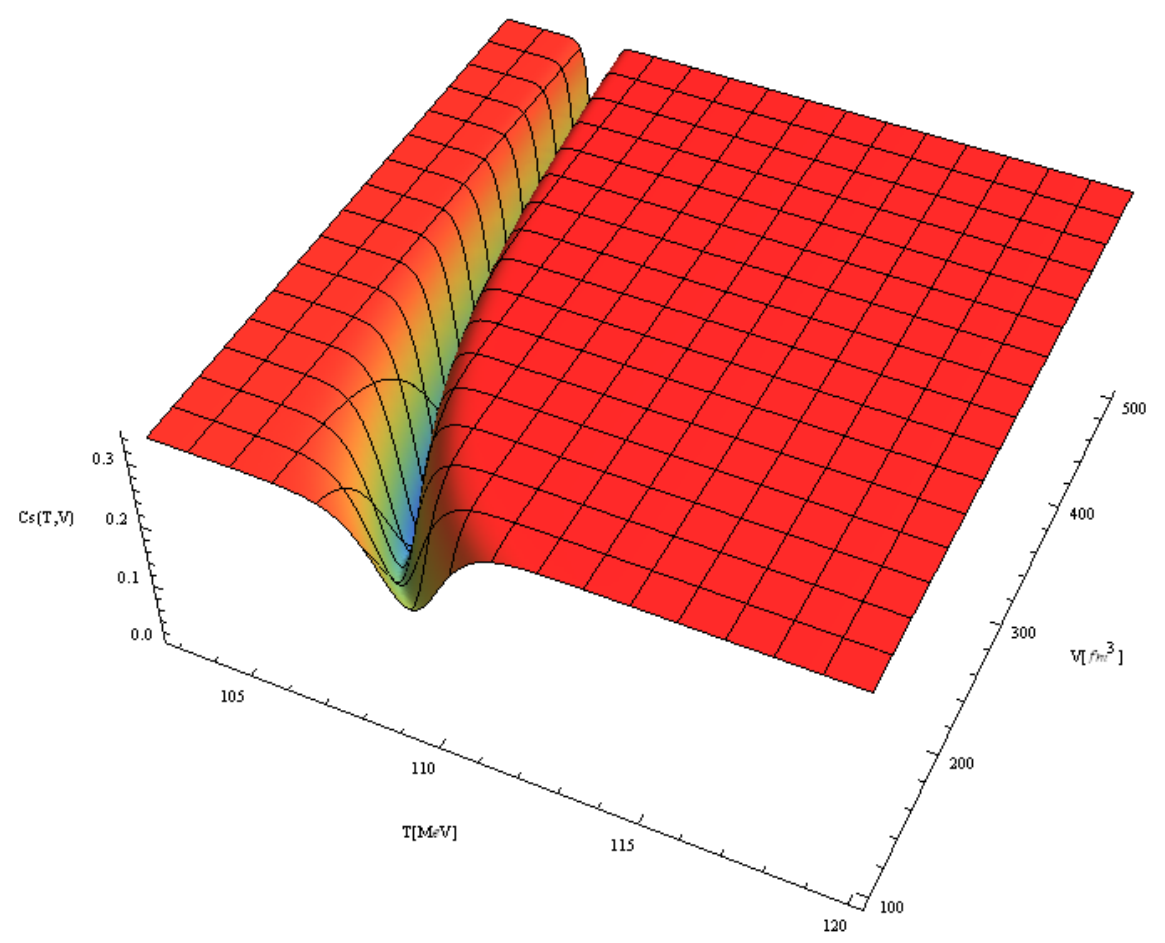

Fig. 5. 3-dim sound velocity $\mathscr{C}_{s}^{2}(T, V)$ of the system as a function of $(T, V)$.

\subsection{Two correlation forms: Results and discussions}

The EoS provides direct insight into the relevant degrees of freedom and their correlation in different phases of the system. The study of EoS by calculating some TRF as functions of $T$ is not the only possibility. Another way consists to eliminate the temperature variable and to replace it by the energy density and obtain for example the pressure as a function of the energy density. So, it is of importance to eliminate the temperature variable in favor of a correlation between the pressure $\mathscr{P}$, the ratio $\mathscr{P} / \epsilon$ and the sound velocity $\mathscr{C}_{s}^{2}$ as a function of energy density $\epsilon$. This can reveal us much more about the EoS than the first. In the context of our Colorless QCD MIT-Bag Model, two relevant correlations $\frac{\mathscr{P}}{\epsilon}(\epsilon)$ and $\mathscr{C}_{s}^{2}(\epsilon)$ are calculated and studied intensively.

\subsubsection{First form: $\frac{\mathscr{P}}{\epsilon}=\frac{\mathscr{P}}{\epsilon}(\epsilon)$}

It has been noted that in our model with a single component $\mathrm{HG}$, both $\mathscr{P} / \epsilon$ and $\mathscr{C}_{s}^{2}$ exhibit a minimum near the phase transition point, as displayed clearly in Figs. 6 and 7. In other words, the EoS in our colorless QCD MIT-Bag Model is getting softer near $T_{0}(V)$, and the system evolves slower at the minimum of the $\mathscr{P}(T, V) / \epsilon(T, V)$ ratio. This minimum is known as the softest point of the EoS. As can be seen 


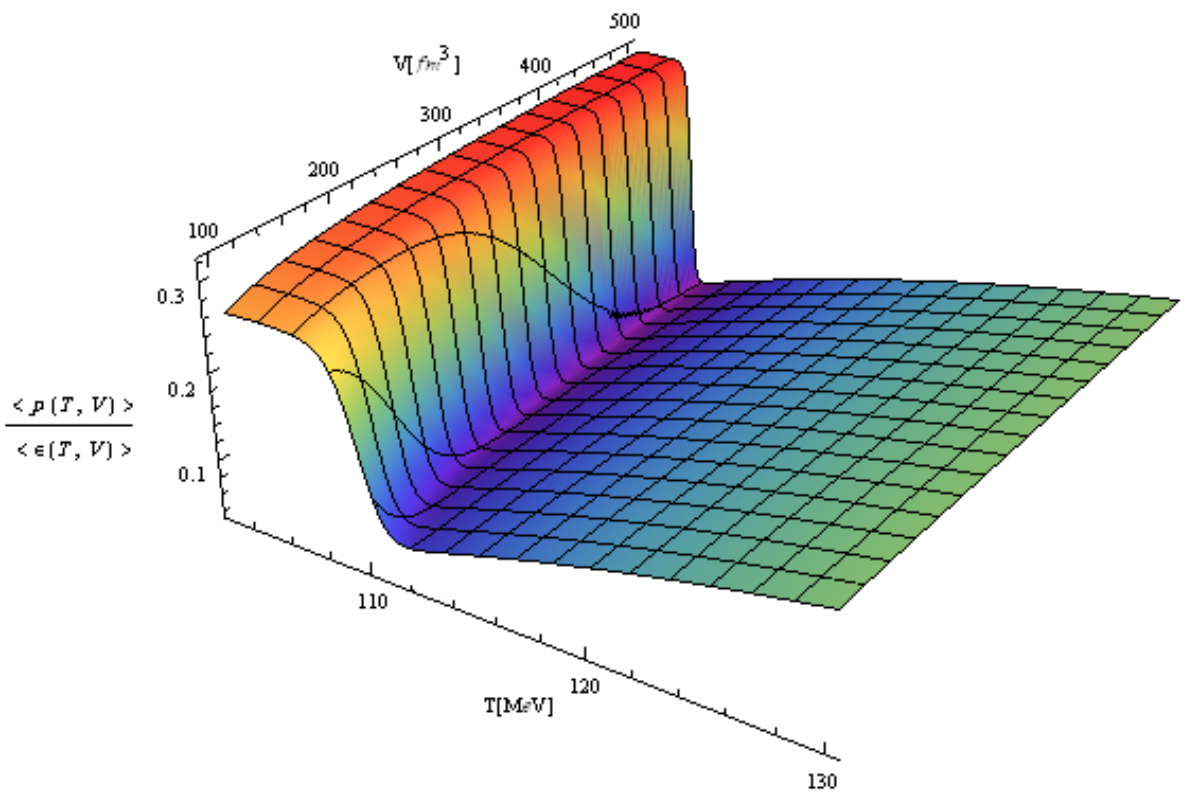

Fig. 6. 3-dim plot of $\mathscr{P} / \epsilon(T, V)$ of the system as a function of $(T, V)$.

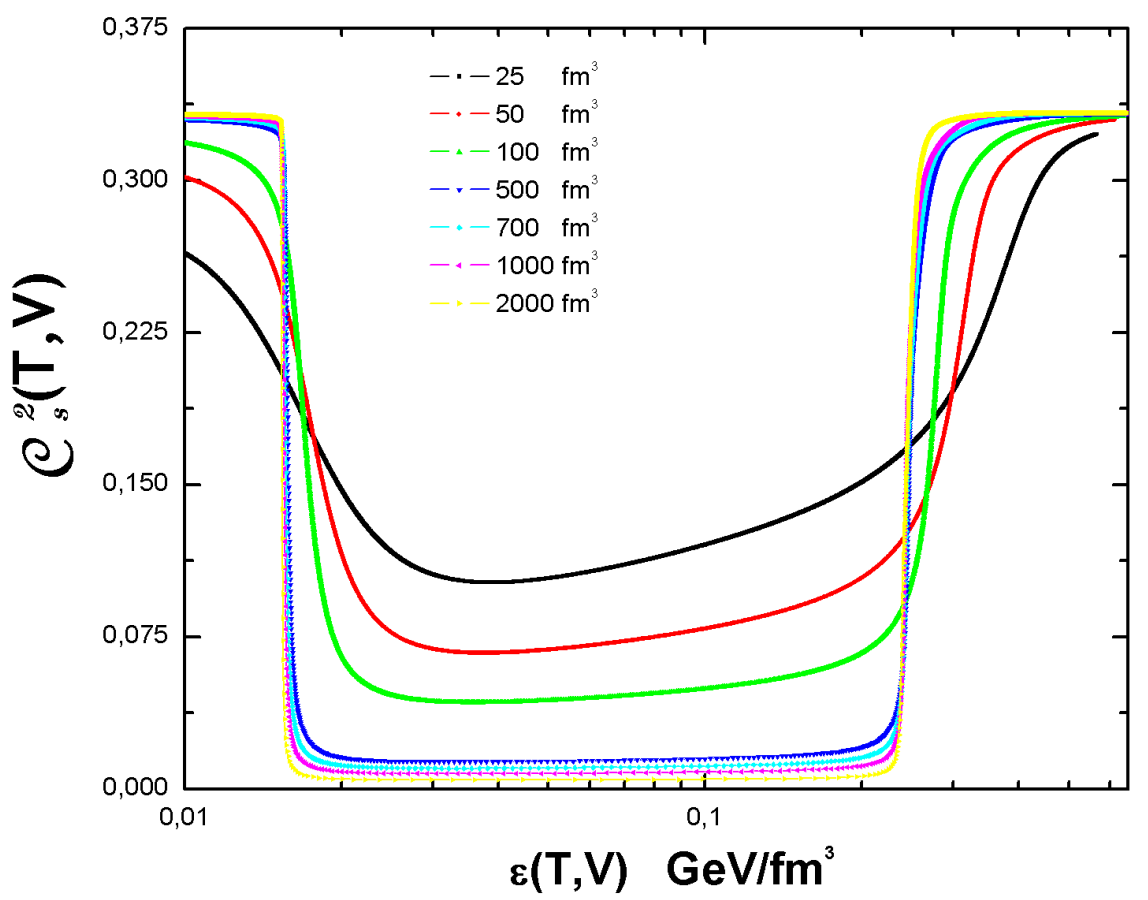

Fig. 7. Sound velocity $\mathscr{C}_{s}^{2}(T, V)$ of the system versus energy density $\epsilon(T, V)$. 
at low temperature and high temperature that the ratio $\mathscr{P} / \epsilon$ versus $\epsilon$ shows little dependence on the volume. In finite volumes, the ratio $\mathscr{P} / \epsilon$ begins to decrease with the increase in energy density until reaching the softest point. Beyond this point, the ratio changes the direction of its variation and increases with the energy density. It is argued that at this point the smallness of the pressure prevents a fast expansion and cooling of the system, it corresponds to a slow-burning regime. As the volume decreases, the minimum of the ratio increases and the softening of the equation of state is less pronounced. On a range of energy density the pressure remains constant meaning that the system does not perform mechanical work. This region is referred to as the softest region of the EoS in which a clear finite volume effect is observed. We also note that for large volumes, the initial value of the ratio in the pure HG phase is exactly the same as the asymptotic value, reached far beyond the softest point in the pure phase of CPP. When the deconfinement phase transition from the hadronic matter to the CPP occurs, the change in pressure is considerably weaker, leading thus to a small sound velocity which is given by the pressure gradient with respect to energy density. This soft region in EoS of our system is expected to have a significant influence on the collective dynamics of the hot and dense matter formed in URHIC. In particular, a small sound velocity delays the expansion of the compressed matter and also leads to a reduced transverse collective flow. The softened EoS leads to both a minimum in the incident energy dependence of the transverse collective flow and a delayed expansion of the compressed matter as in the hydrodynamical model. The softest point of the $\operatorname{EoS}$ is found to be $(\mathscr{P} / \epsilon)_{\min } \simeq 0.03$ at $V=1000 \mathrm{fm}^{3}$ and $(\mathscr{P} / \epsilon)_{\min } \simeq 0.07$ at $V=100 \mathrm{fm}^{3}$, and this is in agreement with what it was found in lattice QCD. ${ }^{27,28}$

A simple proof of the ansatz (12). In the context of our model, it is very easy to formulate a logical and physical justification of the ansatz (12). Indeed, starting from the relation $(47)$

$$
\frac{\mathscr{P}}{\epsilon}(T, V)=\frac{1}{3}\left(1-\frac{4 \mathfrak{B} \mathscr{Q}(T, V)}{\epsilon(T, V)}\right),
$$

and using the asymptotic expressions of $\mathscr{Q}(T, V), \mathscr{H}(T, V)$ and $\epsilon(T, V)$ in the hightemperature limit, in which we have only a pure CPP,

$$
\left\{\begin{array}{l}
\lim _{T \rightarrow \infty} \mathscr{Q}(T, V) \simeq 1, \\
\lim _{T \rightarrow \infty} \mathscr{H}(T, V) \simeq 0, \\
\lim _{T \rightarrow \infty} \epsilon(T, V) \simeq \mathbf{a}_{\mathrm{QG}} T^{4},
\end{array}\right.
$$

a result in complete agreement with the hot QCD's prediction stating that at high temperature and/or high chemical potential the PP behaves like an ideal gas. The QCD nonperturbative effects, which are mainly embodied in the Bag constant, must disappear completely at high temperature, meaning that the bag pressure should 
decrease with the temperature: $\lim _{T \rightarrow \infty} \mathfrak{B}(T)=0.30,31$ Therefore, one can easily rewrite the relationship (50) in a convenient form,

$$
\frac{\mathscr{P}(T, V)}{\epsilon(T, V)}=\frac{1}{3}\left(1-\frac{4}{1+\mathfrak{B}^{-1} \epsilon}\right)
$$

in which the energy density $\epsilon$ represents only the pure CPP contribution.

We can see that this expression has exactly the same form of the parametrization used (12), so we should not consider this parametrization as an ansatz. As we know, the nonperturbative behavior of the vacuum in QCD cannot only be represented by a nonvariable Bag constant. At high temperature, corrections due to the finite temperature effect in the Bag constant must be taken into account and consequently the pressure, energy density and speed of sound must be modified. Unfortunately, as we see in (50) the corrections at high temperature generated by this parametrization are proportional to $T^{4}$, which are completely different from those obtained from the perturbative calculations vanishing only logarithmically as a function of $T$. So, for a more realistic parametrization, the inclusion of thermal effects in the Bag constant $\mathfrak{B}(T)$ is necessary. However, this only confirms that the origin of the confinement is probably much more complicated as that, it could be described by the simple bag model.

\subsubsection{Second form: $\mathscr{C}_{s}^{2}=\mathscr{C}_{s}^{2}(\epsilon)$}

The speed of sound has long been considered as a sensitive hydrodynamic function to the behavior of the strongly interacting matter undergoing a variation in its EoS (phase transition). Early lattice QCD studies found the expected sharp dip of $\mathscr{C}_{s}^{2}$ in the transition region as obtained in our 3-dim plot in Fig. 5. Later, precise studies seemed to indicate a weaker dip, in agreement with the idea that the deconfinement phenomenon is expected to be a rapid cross-over, rather than a real phase transition with a singular behavior. From the definition of $\mathscr{C}_{s}^{2}$ we can derive the following relation:

$$
\mathscr{C}_{s}^{2}=\left(\frac{\partial \mathscr{P}}{\partial \epsilon}\right)=\left(\frac{\mathscr{P}}{\epsilon}\right)+\epsilon \frac{\partial}{\partial \epsilon}\left(\frac{\mathscr{P}}{\epsilon}\right) .
$$

The energy density dependence of $\mathscr{P} / \epsilon$ is related to $\mathscr{C}_{s}^{2}$. In high-temperature region as well as in the finite volume transition region where the derivative vanishes, $\mathscr{C}_{s}^{2}$ is directly given by $\mathscr{P} / \epsilon$. Let us now return to the curves displayed in Fig. 7 that illustrate the variation of the $\mathscr{C}_{s}^{2}$ with the energy density for different volumes. We noticed that in the thermodynamic limit the $\mathscr{C}_{s}^{2}$ takes the ideal gas value $\left(\frac{1}{3}\right)$ in the two regions $\epsilon \leq \epsilon_{\mathrm{HG}}$ and $\epsilon \geq \epsilon_{\mathrm{CPP}}$. On a range of energy density between $\epsilon_{\mathrm{HG}}$ and $\epsilon_{\mathrm{CPP}}$, i.e. in the mixed phase, the speed of sound is nearly vanishing, reflecting the first-order character of the transition. In our case and from plots displayed in Fig. 7, we can extract the numerical values of $\epsilon_{\mathrm{HG}} \sim 0.015 \mathrm{GeV} \mathrm{fm}^{-3}$ and $\epsilon_{\mathrm{CPP}} \sim$ $0.240 \mathrm{GeV} \mathrm{fm}^{-3}$. It has been interpreted that the state of the mixed phases does not expand due to its internal pressure, even if there are strong gradients in the energy density, which has the consequence of not performing mechanical work and therefore 
cools less quickly. Thus, the expansion of the system is delayed and its lifetime is considerably prolonged. For small systems, the sound velocity is damped in a larger domain of energy density and does not vanish, since pressure gradients are finite, but they are still smaller than for an ideal gas equation of state, and therefore the tendency of the system to expand is also reduced. We also find, when approaching the thermodynamical limit, that the sound velocity, in the CPP phase, $\mathscr{C}_{s}^{2}$ reaches the ideal gas value $\mathscr{C}_{s}^{2}=1 / 3$ for the energy densities values less than in the finite volume case. This what it is displayed in Fig. 7. The minimum in the sound velocity is found to be $\left(\mathscr{C}_{s}^{2}\right)_{\min } \simeq 0.007$ at $V=1000 \mathrm{fm}^{3}$ and $\left(\mathscr{C}_{s}^{2}\right)_{\min } \simeq 0.042$ at $V=$ $100 \mathrm{fm}^{3}$. Here, we also noticed that an agreement with the result obtained in lattice QCD. ${ }^{27,28}$ The sound velocity value drops by a factor of 7 at $V=1000 \mathrm{fm}^{3}$ and by a factor of 3 at $V=100 \mathrm{fm}^{3}$ when the density energy is between the two limits.

\section{Fitting Results, Comparisons and Discussions}

After discussing our results on colorless QCD EoS extensively, and in order to complete their interpretation and give them a physical meaning, a comparison work between them and those obtained from different parametrizations based on other models is carried out. Once the parameters of fit models are determined to reproduce our results, we can use them to compute quantities that cannot be evaluated directly, such as the strong coupling constant $\alpha_{s}(T)$, the plasma parameter $\Gamma_{\mathrm{CPP}}$ and some hydrodynamical quantities. The numerical values of the different parameters obtained from the fitting analysis are summarized in Tables 1-4. As can be seen, the results are homogeneous and fluctuate around mean values by a few percents. We also observed their sensitivity to the finite volume effects. Such effects, which we intend to study with details in a forthcoming work. We summarize the most relevant points of this comparison.

Tables 1 and 2, contain the numerical values of the parameters obtained from the two first parametrizations 4 and 7 . First, the numerical value of $\mathbf{B}_{\mathbf{2}}$, which corresponds to the Bag constant, is consistent with the used numerical value $\mathfrak{B}^{1 / 4}=145 \mathrm{MeV}$. Only a finite volume effect is noticed since its value decreases with increasing of the volume. However, the parameter $\mathbf{A}_{\mathbf{2}}$ representing the pdof and has a numerical value which is compatible with $\mathbf{a}_{\mathrm{QG}}=4.0575$ given in (24). We notice that the mean value of $94.829 \mathrm{MeV}$ of the parameter $\mathbf{D}_{\mathbf{1}}$, which represents the QCD-scale parameter, is consistent with the results obtained in the context of the self-consistent quasiparticle model and in the context of the strongly coupled QGP. ${ }^{42,43}$ If we take into account the thermal effects by using the thermal strong coupling constant $\alpha_{s}(T)$, which decreases as the temperature increases, we then get a smaller value of $\Lambda$. From Table 2, it can be noticed that the numerical values of the parameter, $\mathbf{C}_{\mathbf{2}}$ are higher than 0.47 as given by the relation (6) used in the Liquid Partonic Plasma Model. The numerical value of the strong coupling constant $\alpha_{s}\left(T_{0}\right)$ during the deconfinement phase transition, lies within the range of 0.463746 to 0.591666 , consistent with the commonly accepted values. $\stackrel{44}{ }$ It seems as if the 
Table 1. Fitting parameters obtained from Hot QCD parametrization.

\begin{tabular}{ccccc}
\hline System volume $V\left[\mathrm{fm}^{3}\right]$ & $A_{1}$ & $B_{1}$ & $C_{1}$ & $D_{1}^{-1}=\Lambda[\mathrm{MeV}]$ \\
\hline 100 & 5.5824 & 1.7340 & 0.3853 & 93.8293 \\
500 & 5.2696 & 1.3723 & 0.2963 & 96.5168 \\
1000 & 5.3568 & 1.4882 & 0.3245 & 94.4868 \\
10000 & 5.3568 & 1.4882 & 0.3245 & 94.4868 \\
\hline
\end{tabular}

Table 2. Fitting parameters obtained from Liquid Partonic Plasma Model parametrization.

\begin{tabular}{ccccc}
\hline System volume $V\left[\mathrm{fm}^{3}\right]$ & $A_{2}=a_{\mathrm{QG}}$ & $B_{2}=\mathfrak{B}^{1 / 4}$ & $C_{2}$ & $D_{2}[\mathrm{MeV}]$ \\
\hline 100 & 4.1334 & 144.285 & 0.470301 & 4.31574 \\
500 & 4.0895 & 142.262 & 0.463746 & 8.54853 \\
1000 & 4.0962 & 140.588 & 0.591666 & 7.36869 \\
10000 & 4.0790 & 141.246 & 0.529831 & 8.29141 \\
\hline
\end{tabular}

Table 3. Parameters obtained from the Bannur's parametrization.

\begin{tabular}{ccccccc}
\hline System volume $V\left[\mathrm{fm}^{3}\right]$ & $A_{3}$ & $B_{3}$ & $C_{3}$ & $D_{3}$ & $E_{3}$ & $F_{3}$ \\
\hline 100 & 5.888 & 0.096 & 0.023 & 49092.2 & 0.695 & 0.002 \\
500 & 5.838 & 0.135 & 0.023 & 43483.4 & 0.635 & 0.004 \\
1000 & 5.815 & 0.159 & 0.024 & 42786.9 & 0.639 & 0.004 \\
10000 & 5.810 & 0.160 & 0.024 & 42697.6 & 0.639 & 0.004 \\
\hline
\end{tabular}

Table 4. Parameters obtained from the Lattice QCD parametrization

\begin{tabular}{ccccc}
\hline System volume $V\left[\mathrm{fm}^{3}\right]$ & $A_{4}$ & $B_{4}$ & $C_{4}$ & $D_{4}$ \\
\hline 100 & 0.69259 & 1.18691 & 1.35761 & 2.06881 \\
500 & 0.87645 & 1.68099 & 1.51914 & 1.98925 \\
1000 & 0.77351 & 1.21917 & 1.16610 & 1.97172 \\
10000 & 0.83270 & 1.57519 & 1.57007 & 1.81430 \\
\hline
\end{tabular}

$\alpha_{s}(T)$, just beyond the transition point, increases with temperature, a behavior in complete agreement with the appearance of a strongly interacting CPP, even a more strongly interacting system than expected from the Liquid Partonic Plasma Model. It is clear that in the interesting region around the finite volume transition point, the strong coupling should be large as a consequence of the important role played by the nonperturbative effects. This means that the character of ideality does not manifest itself immediately after the colorless deconfinement because the system becomes, somehow, a cradle where the different partons interact strongly in the transition region.

The careful analysis of the obtained fits showed us that three terms of the perturbative expansion of the hot QCD pressure (4) are not sufficient to correctly describe 
the effects of confinement and colorlessness condition (Fig. 8). The Bag constant term and the colorlessness condition provides further measures for nonperturbative physics which cannot be absorbed into the second and third terms in the perturbative expansion (4). On the other hand, when we use only two perturbative terms in addition to the term containing the Bag constant the description of the pressure beyond the transition point becomes very well (5). The Liquid Partonic Plasma Model contains all these ingredients and providing a correct model for our CPP, which appears more like a liquid than a gas (Fig. 9). This is in complete agreement with the experimental observations. $\frac{49}{-52}$ The problem of confinement subsists and its consideration remains a manual action as an ad hoc term, becoming a necessary term in the EoS. Nevertheless, it seems that a more detailed analysis of the hightemperature behavior and a better control of the fit parameters is needed in order to establish a relation to the thermal perturbative pressure.

We notice that the Bannur's pressure does not describe our results well (Fig. 10) compared to the first two models (Fig. 10). Indeed, it underestimates our pressure for small $T$ values and again for large $T$ values. However, there is an overestimation for medium $T$ values. This type of deviations generates a nonrandom behavior in the corresponding residuals. From the analysis of the standardized residuals of each fit, we noticed that they oscillate around the zero value in the case of the hot QCD model and the Liquid Partonic Plasma Model than in the Bannur's model (10). This confirms the excellent goodness-of-fit using hot QCD and Liquid Partonic Plasma Models. The advocated error-statistical arguments by comparing the residuals of hot QCD, Liquid Partonic and Bannur's pressures, showed that the formers are statistically adequate, but the latter is not. The smallness of the fit residuals is merely a diagnostic tool and with their oscillating shape can, therefore, be considered as a signal for the goodness-of-fit. $\underline{45}$

Probably, the insufficiency of the Bannur's model, in describing our results (Fig. 10) is due in the fact that the final expression of the pressure (9) was derived mathematically without the contribution of the $q-g$ and $\bar{q}-g$ interactions and which requires obvious corrections that can lead to a completely different mathematical relationship. When part of partonic interactions is off, logically there is a an overestimate of the pdof, given by (11). That is exactly what we found in our case, an average value of the pdof equal to 5.667. This effect is in complete agreement with the famous result, obtained by Dashen et al., $\frac{46}{4}$ showing that the thermodynamics of a hadronic interacting gas is equivalent to the thermodynamics of a gas of free hadrons and their respective resonances. Therefore, we expect that a refined Bannur's model, taking into account all multiparton interactions, would better describe the results of our EoS.

At the conclusion of this first part of discussion, it is evident to say that the hot QCD Model (Fig. 8) and the Liquid Partonic Plasma Model (Fig. 9) fits are correct and quite similar, but the $\chi^{2}$ obtained in the second model is the best one as displayed in Fig. 9. 


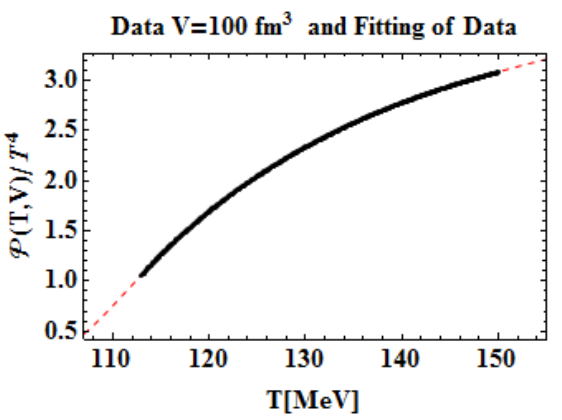

Data $V=10^{3} \mathrm{fm}^{3}$ and Fitting of Data

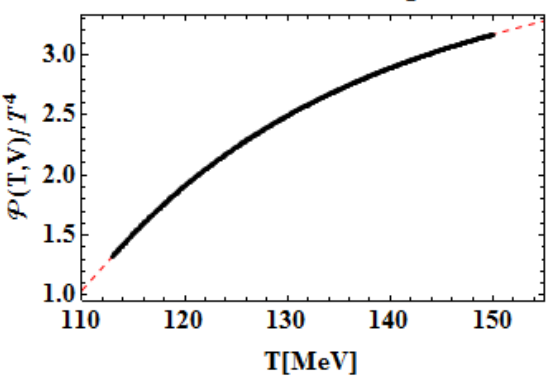

Data $V=500 \mathrm{fm}^{3}$ and Fitting of Data

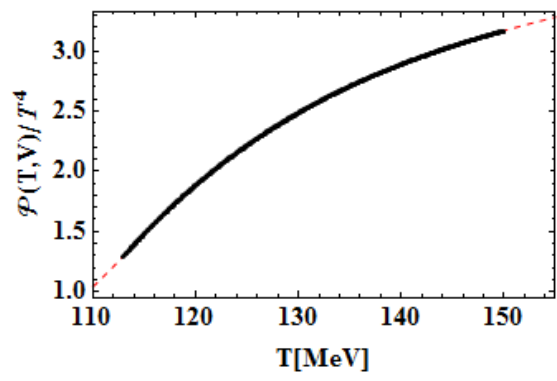

Data $V=10^{4} \mathrm{fm}^{3}$ and Fitting of Data

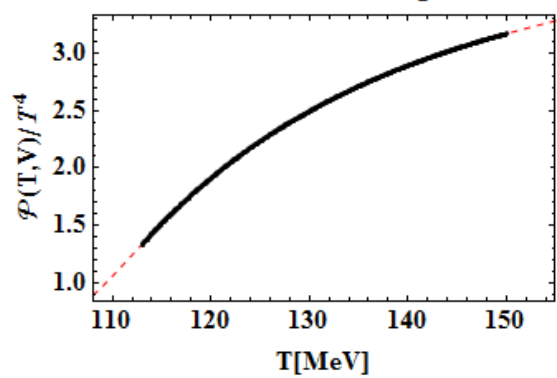

Fig. 8. Fitting of $\left(\frac{\mathscr{P}(T, V)}{T^{4}}\right)$ using $\left(\frac{\mathscr{P}_{1}(T)}{T^{4}}\right)$ versus temperature (hot QCD).
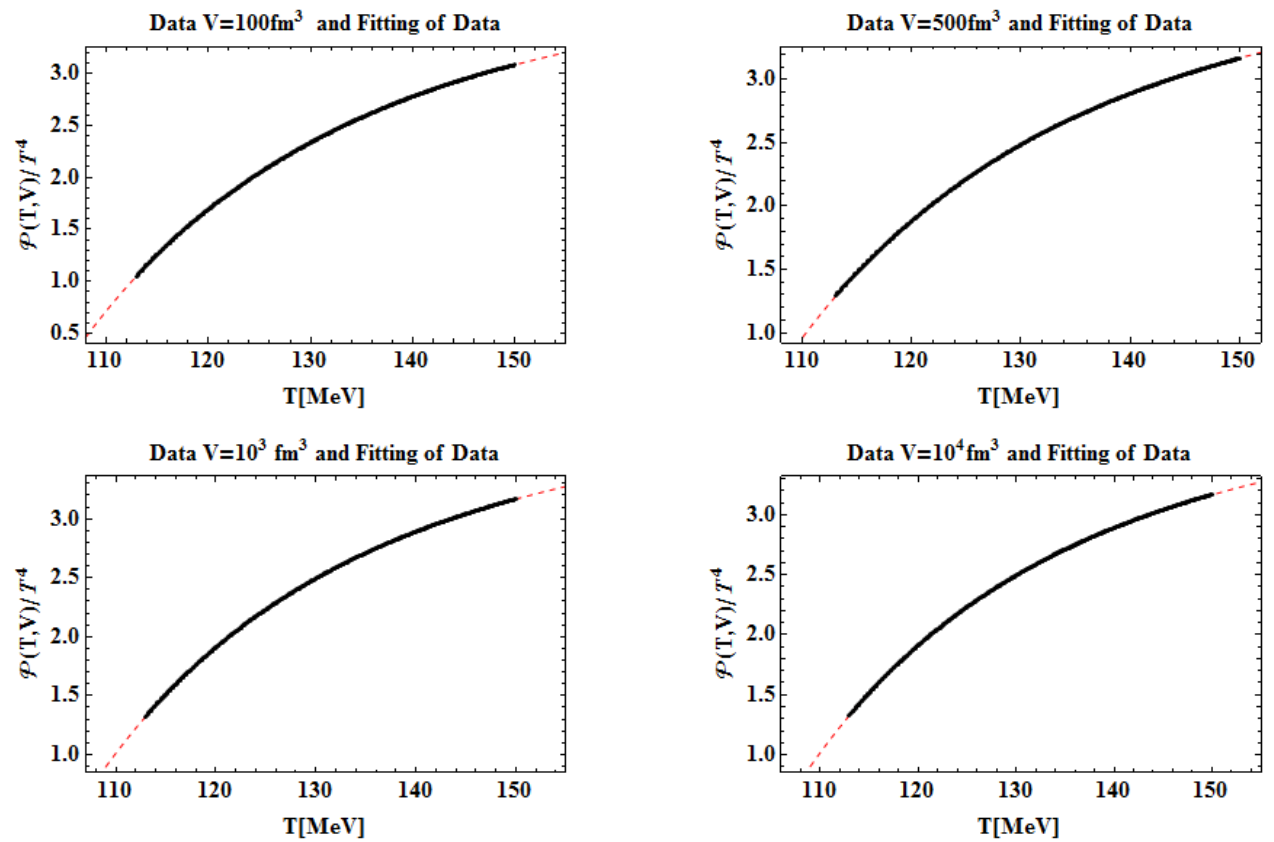

Fig. 9. Fitting of $\left(\frac{\mathscr{P}(T)}{T^{4}}\right)$ using $\left(\frac{\mathscr{P}_{2}(T)}{T^{4}}\right)$ versus temperature (Liquid Partonic Plasma Model). 
Fitting Our Results at $\mathrm{V}=100 \mathrm{fm}^{3}$

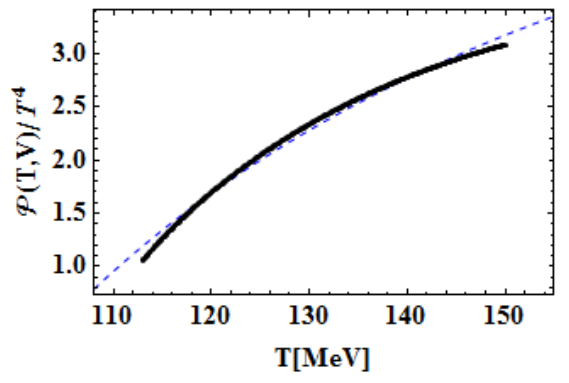

Fitting Our Results at $\mathrm{V}=10^{3} \mathrm{fm}^{3}$

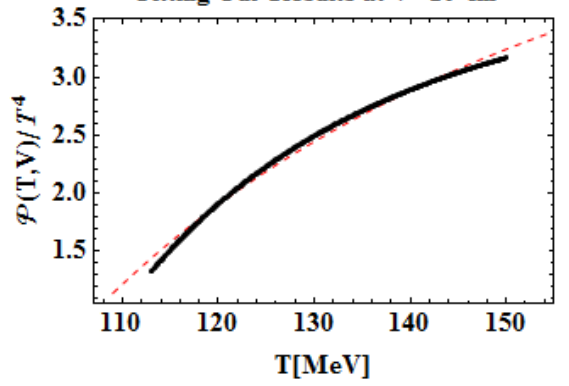

Fitting Our Results at $\mathrm{V}=500 \mathrm{fm}^{3}$

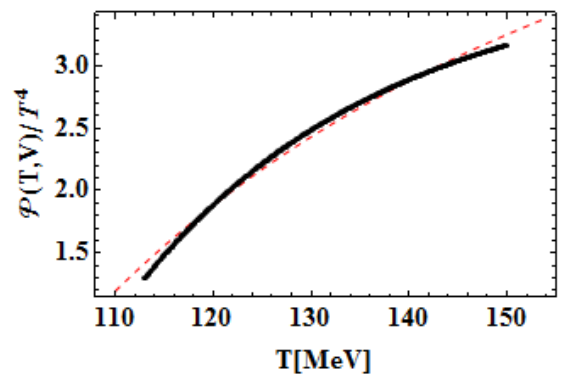

Fitting Our Results at $\mathrm{V}=10^{4} \mathrm{fm}^{3}$

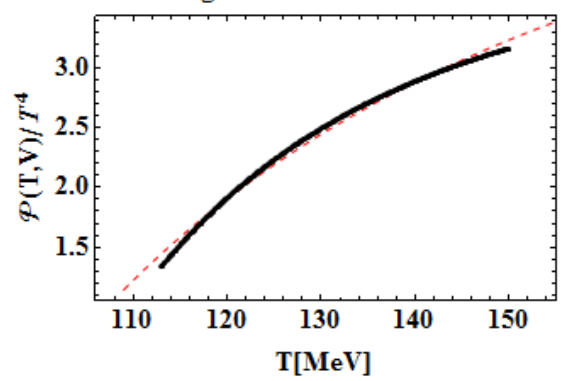

Fig. 10. Fitting of $\left(\frac{\mathscr{P}(T, V)}{T^{4}}\right)$ using $\left(\frac{\mathscr{P}_{3}(T)}{T^{4}}\right)$ versus temperature (Bannur's model).
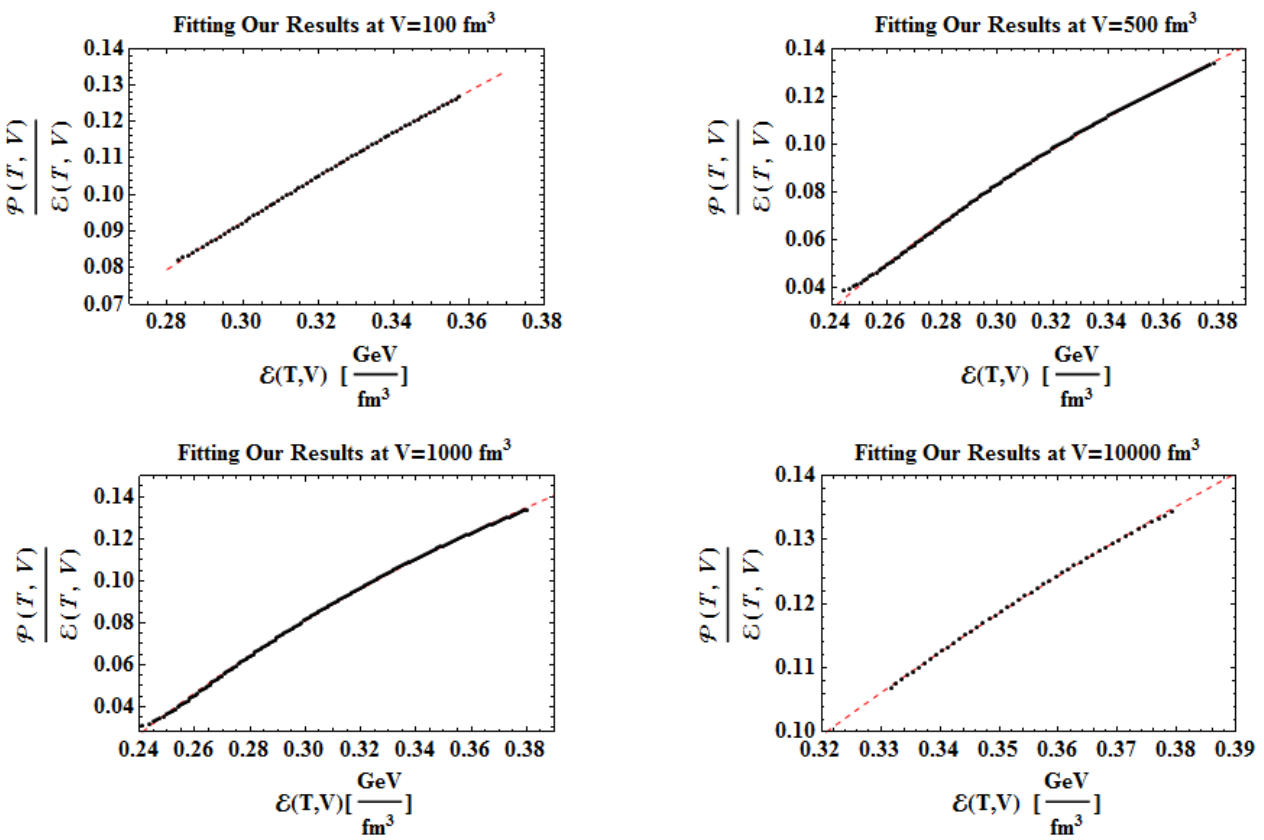

Fig. 11. Fitting of $\left(\frac{\mathscr{P}}{\epsilon}\right)(\epsilon)$ using $\left(\frac{\mathscr{P}}{\epsilon}\right)_{4}$ versus energy density. 


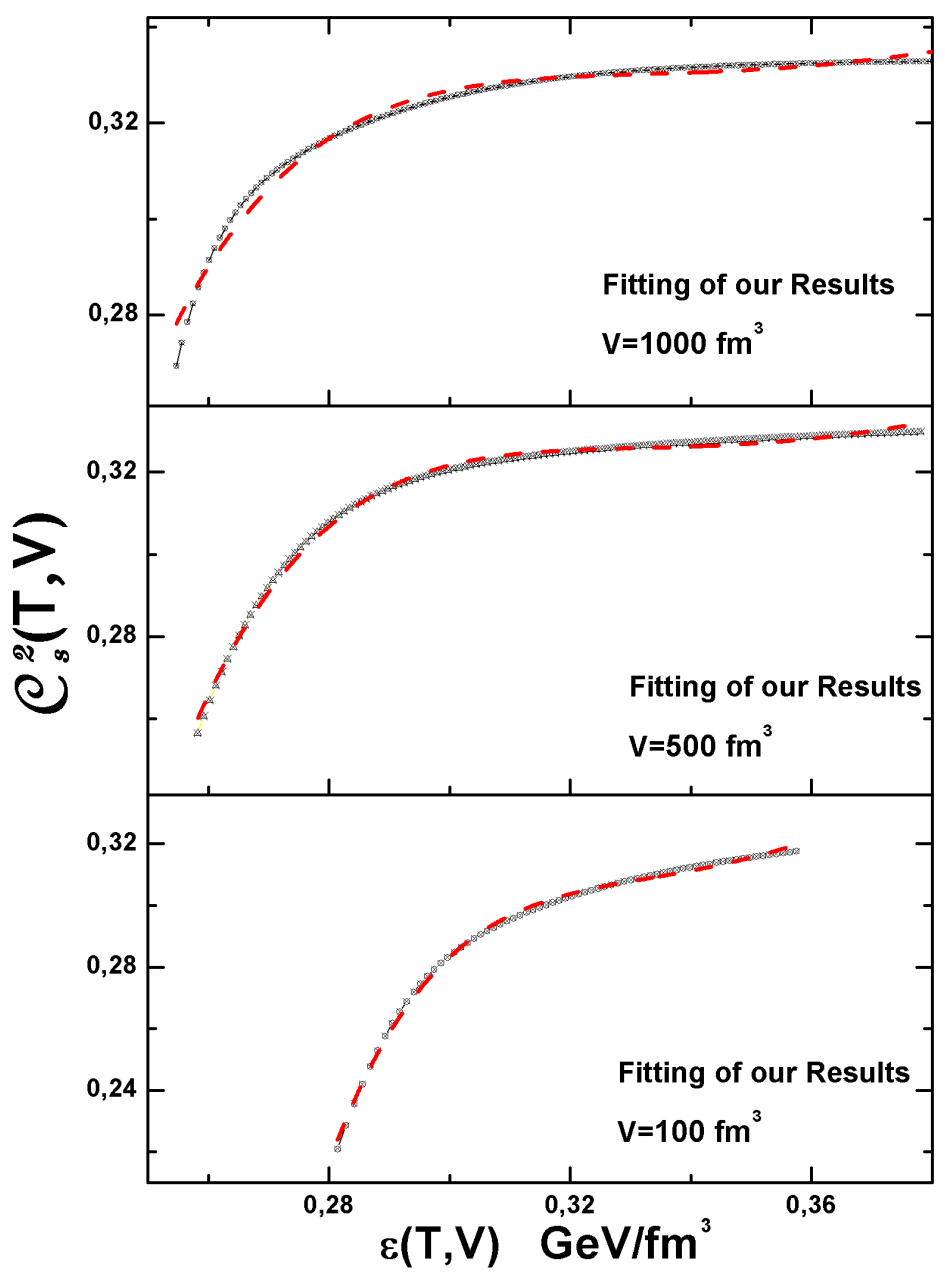

Fig. 12. Fitting of sound velocity $\mathscr{C}_{s}^{2}(\epsilon)$ using lattice QCD parametrization $\left(\mathscr{C}_{s}^{2}(\epsilon)\right)_{4}$.

This proves that the Liquid Partonic Plasma Model (8) is more appropriate than the hot QCD model (4) and even more than the Bannur's model (10). It reveals that the expression of the strong coupling used in the context of Liquid Partonic Plasma Model (6) is more appropriate than in the Hot QCD (2), since only the high temperature approximate expression of the Liquid Partonic Plasma pressure (8) is similar to that of the hot QCD pressure (4).

Now, considering the $\mathscr{P}(\epsilon) / \epsilon$ ratio and sound velocity $\mathscr{C}_{s}^{2}(\epsilon)$ as functions of energy density $\epsilon$, our results are very well described by the two parametrizations of (12) and (13) as displayed by Figs. 11 and 12. As was noted above, for CPP

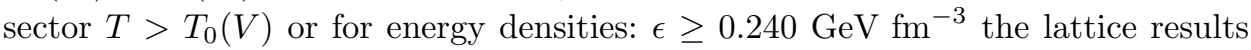
predict the same values of the $\mathscr{C}_{s}^{2}$ and $\mathscr{P} / \epsilon$, as compared to our results. As can 
be seen, our CPP EoS matches very well with the first QCD-EoS containing three massive quarks (two light quarks and a heavy strange quark), $\frac{28}{2}$ and also with the second QCD EoS containing two massive light quarks. ${ }^{27}$ From the numerical values given in Table 4, the first three fit parameters are similar and only the fourth one is different (see Refs. 27 and 28). This can be interpreted as the result of the mass effect and the strange flavor effect. Since our calculation is performed only with two massless quarks and massless pions. Using the more recent lattice QCD results reported in Refs. 29 and 32, performing the fit with the same parametrization (12), once again a good agreement is obtained. Moreover, we have also tried the same fit with only three parameters, the third parameter being fixed: $C_{4}=1$, the quality of fit is better and the numerical values of the remaining parameters become very similar with those extracted from lattice QCD models. ${ }^{29,32}$

Finally, in our estimate of the color-Coulomb coupling parameter of the CPP as defined in Refs. 39, 40, 44, 47, 48, in analogy with that of the QED plasma, and assuming equal (color) magnetic and electric potential energies, one gets

$$
\Gamma_{\mathrm{CPP}}=\frac{8 \pi}{3} \sqrt[3]{\frac{2 \pi \mathbf{a}_{\mathrm{QG}}}{3}} \alpha_{S}(T),
$$

corresponding to the following numerical values

$$
7.92 \leq \Gamma_{\mathrm{CPP}} \leq 10.10 .
$$

Consequently, these values show that our CPP can be widely considered to be in a nonideal liquid phase as claimed by some recent experimental and theoretical results (see Refs. 7, 8, 49-52). Moreover, with the increase of the temperature, the plasma parameter becomes smaller and smaller, so it can be expected that the CPP will undergo a liquid-gas phase transition at high temperature.

\section{Conclusion}

The thermodynamics of a system undergoing the QCD deconfinement phase transition exhibit in different temperature intervals, quite different physics. This is a part of the richness of the hot QCD theory. So, we can distinguish three intervals in temperature: (1) the low-temperature regime, the physics of the system is described by the hadronic resonance gas model, whose thermodynamic properties are controlled by chiral symmetry breaking; (2) the transition region which is characterized by a genuine nonperturbative physics and presumably is a still strongly interacting matter with a more complicated quasiparticle structure and (3) the high-temperature regime, in which perturbative calculations become valid. The confinement phenomenon concerns any many-parton system and then the colorlessness condition can be considered as an effect of the color interaction between partons rendering the system to be in a colorless state. This manifests itself as a nonideal character in EoS. The ideal gas approximation is quite relevant at high temperatures due to the property of asymptotic freedom in this regime. However, 
during the phase transition phenomenon, when the system is prepared for the colorless deconfinement or just after, things are not so simple, in which the non-Abelian character of the CPP manifests itself in an important manner. Consequently, the consideration of perturbative and nonperturbative corrections of higher order is more than necessary. The careful analysis of the obtained fits showed us that three terms of the perturbative expansion of the hot QCD pressure are not sufficient to correctly describe the effects of confinement and colorlessness condition. On the other hand, when we use only two perturbative terms in addition to the term containing the Bag constant the description of the pressure beyond the transition point becomes better. All models discussed in this work take into account the nonideal character of the partonic system. In our model, this nonideality is introduced through the colorlessness condition considered as an ingredient in the calculation of the total partition function. The good quality of our fits indicates that our results agree very well with the four different models analyzed in this work. Therefore the differences appear in the small details. Of course, to understand the values of the fitting parameters, one needs much more general analytic theory based on hot QCD. However and relatively speaking, the model that best describes our results is the Liquid Partonic Plasma Model, in which the Bag constant term is revealed important. This model contains all necessary ingredients to provide a correct model for our CPP, which appears more like a liquid than a gas. This is in complete agreement with the results obtained from both URHIC, lattice QCD, and other models. Collective phenomena emerging from different partonic interactions in many body system, as a CPP, induce strong correlations that give rise to a long-range order, of liquid-like or crystalline structures and collective dynamic properties. $\stackrel{44,47}{ }$ The results of this work show that the CPP has a reasonable mechanism for inducing liquid behavior in the system, which is consistent with our estimate of the plasma parameter $\Gamma_{\mathrm{CPP}}$.

\section{Acknowledgments}

This research work is supported in part by the Deanship of Scientific Research at Taibah University (Al-Madinah, KSA). M. L. H. Ladrem would like to dedicate this work in living memory of his daughter Ouzna Ladrem (Violette) died suddenly in March 24, 2010. May Allah has mercy on her and greet her in his vast paradise. M. L. H. Ladrem would also like to sincerely thank Rana Meshal, master's student in physics, for her skillful reading of this paper.

\section{References}

1. J. Collins, Foundation of Perturbative QCD, 1st edn. (Cambridge University Press, 2013).

2. T. Muta, Foundation of Quantum Chromodynamics: An Introduction to Perturbative Methods in Gauge Theories, 2nd edn. (World Scientific, 1998).

3. J. Rafelski (ed.), Melting Hadrons, Boiling Quarks - From Hagedorn Temperature to Ultra-Relativistic Heavy-Ion Collisions at CERN (Springer, 2016). 
4. H. Satz, Extreme States of Matter in Strong Interaction Physics (Springer, 2018).

5. R. Hilfer, Int. J. Mod. Phys. B 7, 4371 (1993).

6. M. Ladrem et al., Eur. Phys. J. C 44, 257 (2005).

7. E. Shuryak, Prog. Part. Nucl. Phys. 62, 48 (2009).

8. E. Shuryak, Rev. Mod. Phys. 89, 035001 (2017).

9. M. Ladrem et al., Eur. Phys. J. C 75, 431 (2015).

10. J. Rafelski and M. Danos, Phys. Lett. B 97, 279 (1980).

11. K. Redlich and L. Turko, Z. Phys. C 5, 201 (1980).

12. L. Turko, Phys. Lett. B 104, 153 (1981).

13. H.-T. Elze et al., Phys. Lett. B 124, 515 (1983).

14. H.-T. Elze et al., Z. Phys. C 24, 361 (1984).

15. H.-T. Elze et al., Phys. Lett. B 179, 385 (1986).

16. H.-T. Elze et al., Phys. Rev. A 33, 1879 (1986).

17. H.-T. Elze et al., Phys. Rev. D 35, 748 (1987).

18. H.-T. Elze, Doktorarbeit, Frankfurt am Main (1985), unpublished.

19. S. Herbadji, Magister thesis in theoretical physics, ENS-Kouba, Algiers, Algeria (2007).

20. J. Rafelski and M. Danos, GSI Report 83 (1983), 83NBSRafDan.pdf.

21. A. Tounsi, J. Letessier and J. Rafelski, Hot Hadronic Matter Theory and Experiment, eds. J. Letessier, H. H. Gutbrod and J. Rafelski (Springer, 1995), p. 105.

22. J. Cleymans, R. V. Gavai and E. Suhonen, Phys. Rep. 130, 217 (1986).

23. M. A. A. Ahmed, Master thesis in theoretical physics, Taibah University, Al-Madinah Al-Mounawwarah, KSA (2014).

24. C. Cohen-Tannoudji, Lectures Notes in Atomic and Molecular Physics (College de France, 1997).

25. R. Feynman, Statistical Mechanics: A Set of Lectures (Benjamin Cummings, 1982).

26. R. Balian, From Microphysics to Macrophysics: Methods and Applications of Statistical Physics, Vol. 1, 2nd edn. (Springer-Verlag, 2007).

27. S. Ejiri et al., Phys. Rev. D 73, 054506 (2006).

28. M. Cheng et al., Phys. Rev. D 77, 014511 (2008).

29. S. Borsanyi et al., J. High Energy Phys. 11, 1 (2010).

30. G. Song, W. Enke and J. Li, Phys. Rev. D 46, 3211 (1992).

31. B. K. Patra and C. P. Singh, Z. Phys. C 74, 699 (1997).

32. A. Bazavov et al., Phys. Rev. D 90, 094503 (2014).

33. J. I. Kapusta and C. Gale, Finite-Temperature Field Theory: Principles and Applications, 2nd edn. (Cambridge University Press, 2006).

34. S. Mogliacci et al., J. High Energy Phys. 12, 1 (2013).

35. C. Zhai and B. Kastening, Phys. Rev. D 52, 7232 (1995).

36. K. Kajantie, M. Laine, K. Rummukainen and Y. Schroder, Phys. Rev. D 67, 10508 (2003).

37. J. Rafelski et al., Phys. Rev. C 62, 064901 (2000).

38. J. Rafelski et al., Phys. Rev. C 67, 031902 (2003).

39. V. M. Bannur, Phys. Lett. B 362, 7 (1995).

40. V. M. Bannur et al., Phys. Rev. C 76, 044908 (2007).

41. A. S. Khvorostukhin et al., Nucl. Phys. A 845, 106 (2010).

42. V. M. Bannur, Phys. Rev. C 75, 044905 (2007).

43. V. M. Bannur, J. Phys. G: Nucl. Part. Phys. 32, 993 (2006).

44. B. A. Gelman, E. Shuryak and I. Zahed, Phys. Rev. C 74, 044908 (2006).

45. A. Spanos, Probability Theory and Statistical Inference, 2nd edn. (Cambridge University Press, 2003). 
46. R. Dashen, S.-K. Ma and H. J. Bernstein, Phys. Rev. 187, 345 (1969).

47. M. Thoma, J. Phys. G: Nucl. Part. Phys. 31, L7 (2005) [Erratum: ibid. 31, 539 (2005)].

48. A. Pishier and W. Cassing, Phys. Rev. Lett. 94, 172301 (2005).

49. K. Adcox et al., Phys. Rev. Lett. 88, 022301 (2002).

50. G. Aad et al., Phys. Rev. Lett. 105, 252303 (2010).

51. K. H. Ackermann et al., Phys. Rev. Lett. 86, 402 (2001).

52. K. Aamodt et al., Phys. Rev. Lett. 105, 252302 (2010). 